\title{
Transcriptome analysis identified a novel 3- LncRNA regulatory network of transthyretin attenuating glucose induced hRECs dysfunction in diabetic retinopathy
}

\author{
Jun Shao ${ }^{1+}$, Yunbin Zhang ${ }^{2 \dagger}$, Guangming Fan ${ }^{3+}$, Yu Xin ${ }^{3^{*}}$ and Yong Yao ${ }^{1 *}$
}

\begin{abstract}
Background: Diabetic retinopathy (DR) is the leading cause of blindness in the working age population. Transthyretin (TTR) showed a significantly decreased concentration in DR patients and exerted a visual protective effect by repressing neovascularization. This work intended to identify long non coding RNAs (IncRNAs) and explore their potential mechanism underlying the protective role of TTR.

Methods: Transcriptome of human retinal endothelial cells (hRECS) treated with low glucose (LG), high glucose (HG) or high glucose with $4 \mu \mathrm{M} T \mathrm{TR}(\mathrm{HG}+\mathrm{TTR})$ was conducted. Differentially expressed IncRNAs, mRNAs and TTR related IncRNAs and mRNA were acquired. Functional annotation and Gene Set Enrichment Analysis were applied to analyse TTR affected pathways and processes. Weighted gene co-expression network analysis (WGCNA) was implemented to obtain hub modules and genes. LncRNA-mRNA regulatory networks were constructed based on cis, trans and competing endogenous RNAs acting mode. QRT-PCR was conducted to validate the expression of IncRNAs in aqueous humor and serum samples from 30 DR patients and 10 normal controls.

Results: RNA-sequencing of hRECs treated with low glucose $(L G)$, high glucose $(H G)$ or high glucose with $4 \mu M T R$ (HG + TTR) was conducted. 146,783 protein-coding transcripts, 12,403 known IncRNA transcripts and 1184 novel noncoding transcripts were characterized. A total of 11,407 differentially expressed mRNAs (DE-mRNAs), 679 differentially expressed IncRNAs (DE-IncRNAs) in HG group versus LG group, 6206 DE-mRNAs and 194 DE-IncRNAs in HG + TTR versus HG group were obtained, respectively. 853 TTR-mRNAs and 48 TTR-IncRNAs were acquired, and functionally involved in cell cycle, apoptosis, inflammation signalling pathway, response to oxidative stress, neovascularization and autophagy. The WGCNA analysis identified a hub module of 133 genes, with the core function of oxidative stress response, angiogenesis, MAPK pathway, cell proliferation and apoptosis. After qRT-PCR validation, a 3-IncRNA regulatory network was proposed. At last, IncRNAs MSTRG.15047.3 and AC008403.3 showed significantly relative higher expression levels in both aqueous humor and serum samples, compared with normal controls, and FRMD6-AS2 was significantly down-regulated.

\footnotetext{
* Correspondence: yuxin@jiangnan.edu.cn; yaoyongchina@outlook.com †Jun Shao, Yunbin Zhang and Guangming Fan contributed equally to this work.

${ }^{3}$ Key Laboratory of Industry Biotechnology, Ministry of Education, School of Biotechnology, Jiangnan University, Wuxi 214122, Jiangsu, China

'Department of Ophthalmology, Wuxi People's Hospital affiliated to Nanjing Medical University, Wuxi 214023, Jiangsu, China

Full list of author information is available at the end of the article
}

(c) The Author(s). 2019 Open Access This article is distributed under the terms of the Creative Commons Attribution 4.0 International License (http://creativecommons.org/licenses/by/4.0/), which permits unrestricted use, distribution, and reproduction in any medium, provided you give appropriate credit to the original author(s) and the source, provide a link to the Creative Commons license, and indicate if changes were made. The Creative Commons Public Domain Dedication waiver (http://creativecommons.org/publicdomain/zero/1.0/) applies to the data made available in this article, unless otherwise stated. 
(Continued from previous page)

Conclusions: TTR regulated mRNAs and biological processes including oxidative stress, inflammation signalling and autophagy. A 3-IncRNA regulatory network was characterized underlying TTR repressing neovascularization, and showed potential diagnostic performance in DR.

Keywords: Diabetic retinopathy, Transthyretin, Transcriptome, 3-IncRNA regulatory network, diagnosis

\section{Background}

As the leading cause of vision impairment and loss in the working age population, diabetic retinopathy (DR) is a common and specific microvascular complication characterized by early retinal microvascular dysfunction [1]. In China, the number of DR patients is growing each year [2]. The early stage of DR is exemplified by retina endothelial cells and pericytes death [3]. As DR progresses, vascular leakage becomes evident and may eventually lead to diabetic macular edema, the most common cause of vision loss in DR [4]. Till now, panretinal photocoagulation, vitrectomy and anti-neovascularization drug intraocular injection remain the few therapeutic options [5]. Accumulating evidence implicated that inflammation, cell apoptosis, oxidative stress and autophagy were major causative factors involved in the pathogenesis of DR [6]. Therefore, uncovering potential mechanisms underlying DR pathogenesis, especially revealing hub genes and pathways in inflammation, cell apoptosis, oxidative stress and autophagy is of paramount importance for the development of effective therapeutics for patients with DR.

Transthyretin (TTR) is a $55-\mathrm{kDa}$ homotetramer protein reported to transport thyroxine and retinol through retinol-binding protein (RBP) [7], and it is mainly secreted by human retinal pigment epithelial cells (hRPECs) and choroid in ocular tissue. In vitreous and serum samples of high myopia patients, TTR showed significantly higher expression compared with healthy donors [8]. Interestingly, the expression of TTR in DR patients was significantly lower, compared with healthy donors $[8,9]$. In our previous research, an exogenous $4 \mu \mathrm{M}$ TTR treatment or endogenous TTR overexpression both repressed blood vessel tube forming capability of hyperglycemic cultured human retinal endothelial cells (hRECs), by attenuating pro-angiogenic genes expression, like Angiopoietin 2 (Angpt2), Vascular Endothelial Growth Factor Receptor 1 (VEGFR1), and Vascular Endothelial Growth Factor (VEGF2) [10, 11]. Additionally, TTR was reported to repress neovascularization by promoting hRECs apoptosis through direct binding to glucose-regulated protein 78 (GRP78) [12]. Surprisingly, the exogenous added TTR efficiently crossed cell membrane and nuclear membrane, and located in both in cytoplasm and nucleus, implicating complex transcriptional and post-transcriptional regulatory role, while the exact mechanism still remains obscure.
Long noncoding RNA (lncRNA) is characterized to be transcripts with a length of more than $200 \mathrm{nt}$ and of no protein-coding capability. Accumulating evidence pointed that lncRNA exerted function in cis by regulating nearby genes, or in trans by modulating distantly located genes [13]. In addition, it's a putative mechanism that lncRNAs may regulate target gene expression via miRNA response element (MRE), known as competing endogenous RNA (ceRNA) [14]. The aberrant expression of lncRNAs in early diabetic retinopathy was first characterized in 2004 [15]. Subsequent studies of individual lncRNAs playing a transcriptional or post-transcriptional regulatory role in DR pathogenesis and progression had been reported, including Maternally Expressed 3 (MEG3) [16], Myocardial Infarction Associated Transcript (MIAT) [17, 18], HOXA Distal Transcript Antisense RNA (HOTTIP) [19], Metastasis Associated Lung Adenocarcinoma Transcript 1(MALAT1) [20, 21], SOX2 Overlapping Transcript (Sox2-OT) [22], antisense non-coding RNA in the INK4 locus (ANRIL) [23] and BDNF Antisense RNA $(B D N F-A S)$ [24]. However, there have been limited studies of IncRNAs in TTR repressing DR neovascularization.

In this study, we proposed that there may exist certain lncRNAs, through which TTR exerts its function of repressing neovascularization in DR. RNA transcriptome of hRECs treated with low glucose concentration of 5.5 $\mathrm{mM}$ (LG), high glucose concentration of $25 \mathrm{mM}$ (HG), and a $25 \mathrm{mM}$ high glucose plus a concentration of $4 \mu \mathrm{M}$ TTR (HG + TTR) was determined for the first time. The impact of high glucose and TTR on hRECs neovascularization was evaluated with tube formation assay. Then differentially expressed lncRNAs and mRNAs that induced by high glucose and TTR were identified, respectively. Functional annotation and network analysis of TTR regulated mRNAs significantly enriched pathways and biological processes in DR. Furthermore, the TTR regulated lncRNAs and mRNAs were predicted, and the lncRNA-mRNA regulatory network in cis, trans and ceRNA was constructed. Finally, after qRT-PCR validation in hRECs, vitreous humor and serum samples, a 3lncRNA centered TTR response coding-non-coding network with potential diagnosis performance was proposed to explain the protective role of TTR, which would provide basis for the future potential application of TTR and lncRNAs in DR diagnosis and treatment. 


\section{Methods}

\section{Cell culture and TTR treatment}

HRECs from donated human retinal tissue were extracted, sub-cultured, and authenticated by Genetic Testing Biotechnology (Shanghai, China) using Short Tandem Repeat (STR) analysis as described in 2012 in ANSI Standard (ASN-0002) by the ATCC Standards Development Organization (SDO). Nineteen short tandem repeat (STR) loci plus the gender determining locus, Amelogenin, were amplified using the commercially available EX20 Kit from AGCU. The cell line sample was processed using the ABI Prism 3130 XL Genetic Analyzer. Data were analyzed using GeneMapper ID v3.2 software (Applied Biosystems). Appropriate positive and negative controls were run and confirmed for each sample submitted. And as detected, eight core STR loci plus Amelogenin revealed that HRECs should be quite similar with HBMEC-2 (human brain microvascular endothelial cell) (Additional files 1, 2 and 3).

HRECs from passage 4 were diluted to $8 \times 10^{4} / \mathrm{mL}$ and incubated in 6-well plates with low glucose (LG) media until confluent. After washing with PBS, the cells were further cultured for $48 \mathrm{~h}$ at $37^{\circ} \mathrm{C}$ in $5.5 \mathrm{mM}$ glucose (low group, LG), $25 \mathrm{mM}$ glucose (high glucose, HG), or 25 mM glucose with $4 \mu \mathrm{M}$ TTR (HG + TTR) media. The concentration of low glucose of $5.5 \mathrm{mM}$ and simulated high glucose concentration of $25 \mathrm{mM}$ were referred to previous studies $[25,26]$. The concentration of TTR of $4 \mu \mathrm{M}$ were referred to Raquel J. Nunes et al's study; the TTR concentration of $1,2,4,8$ and $10 \mu \mathrm{M}$ were examined and $4 \mu \mathrm{M}$ was determined as the final concentration [27]. Cells were collected and total RNAs were extracted from hRECs, using TRIzol reagent (Takara, Dalian, China), according to the manufacturer's instructions.

\section{Tube formation assay}

The basement membrane matrix (BD Biosciences) was placed into the well of 24-well plate, and hardened at $37^{\circ} \mathrm{C}$ for $30 \mathrm{~min} ; 2 \times 10^{4}$ hRECs cells were seeded on each well, and incubated with glucose and/ or TTR at $37^{\circ} \mathrm{C}$ for $24 \mathrm{~h}$. Tube formation was observed using an Olympus IX-73 microscope.

\section{Subjects}

Aqueous humor and serum samples of $30 \mathrm{DR}$ patients and 10 normal controls (patients without diabetes) were obtained from ophthalmology inpatients of Nanjing Medical University Affiliated Wuxi People's Hospital. Aqueous humor of normal controls (patients without diabetes) and DR patients were collected during cataract surgery. Clinic patients' samples processing were the same as we previously reported [9]. Informed consents were signed for enrolled patients and healthy individuals. This study was compiled with principles of the
Declaration of Helsinki and was approved by the Ethics Committee of Nanjing Medical University (2014-62). All clinical samples were obtained from ophthalmology inpatients of Nanjing Medical University Affiliated Wuxi people's Hospital. Aqueous humor sample of DR patients and normal control were obtained from cataract surgery. All samples were stored at $-80^{\circ} \mathrm{C}$.

\section{Transcriptome sequencing and differentially expressed genes (DEGs) identification}

After total RNAs were extracted and collected, cDNA was produced and purified with AMPure XP (Beckman Coulter, Brea, CA, USA) beads. Then PCR was conducted to generate cDNA libraries, and adaptor sequences were removed, followed by low-quality reads filtering. Trinity (http://trinityrnaseq.sourceforge.net/) software and De Bruijn graph algorithm [28] were applied for de novo assembly. At last, unigenes were annotated with BLASTX and BLAST algorithm, by searching GenBank database (http://www.ncbi.nlm.nih.gov/) and NCBI non-redundant (NR) (ftp://ftp.ncbi.nih.gov/blast/db/), respectively. The RNA-seq data had been submitted to GEO repository, with accession number GSE117238.

In this study, we used FPKM (Fragments Per Kilobase of transcript per Million) for transcripts, instead of gene expression, and gene names corresponding to certain transcripts were also shown. For a certain gene, if more than one transcript showed significant differential expression, the gene were considered to be significantly deregulated. Specifically, Student's t-test and fold change (FC) filtering were conducted, and to screen differentially expressed genes (DEGs) between two groups by using $\mathrm{R}$ software limma package. In addition to $P$ value, a Benjamini-Hochberg procedure was conducted for multiple comparisons to control the false discovering rate (FDR) [29]. Significantly deregulated transcripts were defined using the criteria of Fold Change $\geq 2, P \leq$ 0.05 and FDR $\leq 0.05$. Volcano plot were construed by $R$ software ggplot2 package, to show significantly deregulated transcripts and genes. The differential expression analysis was conducted in the transcript level, and transcripts with the converse expression fold-change trend in HG + TTR group versus HG group and HG group versus LG group were named as TTR related DEGs (TTR-DEGs).

\section{Pathway and GO annotation and GSEA analysis}

For differentially expressed genes functional annotation, KEGG pathway and Gene Ontology (GO) annotation and enrichment were conducted, based on Kyoto Encyclopedia of Genes and Genomes (KEGG) (http:// www.genome.jp/kegg/) and Gene Ontology database (http://www.geneontology.org/), with DAVID (The Database for Annotation, Visualization and Integrated 
Discovery v6.8, https://david.ncifcrf.gov/). For GO analysis, biological processes (BP), cellular components (CC) and molecular function (MF) were applied and enriched items were shown, respectively. A Fisher exact test was used to select only significant categories. A Benjamini-Hochberg procedure was conducted for multiple comparisons to control the false discovering rate (FDR) [29]. GO terms with corrected Q value $<0.05$ were considered significant. Significantly enriched pathways and biological processes were displayed with $\mathrm{R}$ software ggplot2 package.

Compared with functional annotation of DEGs with KEGG pathway and GO, function of all detected genes were analyzed with Gene Set Enrichment Analysis (GSEA) [30]. Specifically, annotation file of c2: curated gene sets-all canonical pathways, gene symbols were used to analyze significantly enriched gene sets. The GSEA analysis was applied to extract significant biological processes and pathways, for HG and TTR + HG groups. Gene expression data matrix of detected genes in LG, HG and TTR + HG groups were imported into GSEA software (v2.2.4) and the permutation index were set as 1000 . Then the GS (gene set) score and $P$ value were used, and biological processes or pathways with $P<0.05$ were considered to be significant.

\section{Protein-protein interaction (PPI) network analysis and sub-network construction}

To explore the interaction of TTR-mRNAs, TTRmRNAs lists were first imported into STRING database (https://string-db.org), and generated the protein-protein interaction (PPI) network. When constructing the PPI network, protein-protein interaction pairs with medium confidence of interaction score $>0.4$ were exported and then visualized by using Cytoscape (version 3.4.0). To analyze the topological structure and relationship in PPI network, CentiScaPe was further applied for calculating centrality parameters for each node and finding the most important nodes in a network [31]. At last, Molecular COmplex Detection (MCODE) (http://apps.cytoscape. org/apps/mcode) was applied to find densely connected regions and sub-networks within the main network.

\section{Weighted gene co-expression network analysis (WGCNA) and module detection}

The Weighted gene co-expression network analysis (WGCNA) is a well-known technique used to identify biologically meaningful and functional co-expression modules related to diseases or conditions [32]. In this study, to explore and screen gene expression modules correlated with high glucose and TTR, the gene coexpression network was constructed by the R WGCNA package. The gene expression similarity and softthreshold were calculated using the integrated function (pick Soft Threshold) in the package. When the soft threshold was defined as 10, a module with ME green color of 133 genes showed highest correlation with both glucose and TTR and was functionally annotated in subsequent steps.

\section{Module genes biological pathway and function network in ClueGo}

The function of $133 \mathrm{ME}$ green module genes were annotated and significantly enriched $(P<0.05)$ pathways were labeled in Cytoscape ClueGo and CluePedia. The ClueGo and CluePedia application were developed to create and visualize a functionally grouped network of terms/pathways [33].

\section{LncRNA cis and trans regulatory network construction}

For lncRNA targets identification, cis and trans regulatory network was constructed. To identify cis regulatory relationship, lncRNAs that act on neighboring target genes were investigated. We searched for coding genes $10 \mathrm{~kb} / 100 \mathrm{~kb}$ upstream and downstream of each lncRNA. To identify trans regulatory relationship, lncRNAs and target genes were identified based on their expression levels. We calculated the expression level correlation between lncRNAs and coding genes using custom scripts., and IncRNA-mRNA pairs with correlation coefficient $>0.95$ or $<-0.95$ were extracted for subsequent analysis. At last, the cis or trans regulatory network was shown with Cytoscape.

Competing endogenous RNAs (ceRNAs) network analysis To reveal the roles and interactions of lncRNAs with mRNAs in glucose and TTR treated hRECs, we constructed lncRNA-miRNA-mRNA regulatory ceRNA networks. Potential miRNA response elements were searched for the sequences of IncRNAs and mRNAs, and we identified overlaps in predicted miRNA seed sequence binding sites as well as lncRNAs binding sites in the target mRNA as part of the lncRNA-miRNA-mRNA interaction. The miRNA binding sites were predicted by miRanda (http://www.microrna.org/), while the miRNAmRNA interactions were predicted by Targetscan (http:// www.targetscan.org/). The interaction network was built and displayed using Cytoscape software based on the screening of IncRNA-miRNA-mRNA pairs.

\section{QRT-PCR}

Cells were collected and total RNAs were extracted from hRECs cells and clinical samples using TRIzol reagent (Takara, Dalian, China), according to the manufacturer's instructions. Aqueous humor and serum samples were centrifuged at $\left(12,000 \mathrm{~g}\right.$ for $10 \mathrm{~min}$ at $\left.4{ }^{\circ} \mathrm{C}\right)$ and pellets were collected for RNA extraction and purification. Briefly, cDNA was synthesized by M-MLV reverse 
transcriptase (Invitrogen, Carlsbad, CA, USA) from extracted RNAs. RT-PCR was performed in triplicate with SYBR Green Real-Time PCR Master Mixes (Takara) on an ABI 7900 PCR system (Applied Biosystems, Bedford, CA, USA). As LncRNA AC008403.3 was co-localized with LMTK3 in chromosome 19 and FRMD6-AS2 and FRMD6 were localized in DNA+/- strands, with partial sequences overlapping (Additional file 2: Figure S1), transcript specific primers were designed. As previous study pointed that, expression of glyceraldehyde-3phosphate dehydrogenase (GAPDH), one of the widely used house-keeping genes, was involved in high glucose induced retinal pericytes apoptosis [34], we chose Beta-Actin (ACTB) as the house-keeping gene in this study. Information about the primers was listed in Table 1. The RT-PCR program comprised an initial denaturing step at $95^{\circ} \mathrm{C}$ for $5 \mathrm{~min}$, followed by 45 cycles of $95^{\circ} \mathrm{C}$ for $20 \mathrm{~s}, 60^{\circ} \mathrm{C}$ for $20 \mathrm{~s}$, and $70^{\circ} \mathrm{C}$ for $20 \mathrm{~s}$. Relative quantification was performed according to the comparative method $\left(2^{-\Delta \Delta \mathrm{CT}}\right.$; Applied Biosystems User Bulletin 2P/N 4303859).

\section{Statistical analysis}

SPSS 13.0 for windows (Chicago, IL, USA) was used for data analysis, and statistical significance was determined using a T test. The $P$ and $T$ values were calculated. $P<$ 0.05 was considered statistically significant.

Table 1 Primers of genes

\begin{tabular}{|c|c|}
\hline Gene & Primer $\left(5^{\prime}-3^{\prime}\right)$ \\
\hline FRMD6-AS2 & $\begin{array}{l}\text { F: CCTGGAACATTGGAAATAGGC } \\
\text { R: TGGCAGCATTAGAATACAGCA }\end{array}$ \\
\hline AC105345.1 & $\begin{array}{l}\text { F: TTGCAGCCTCCTCATCC } \\
\text { R: AAAGCCTGTTGGTGGTCTC }\end{array}$ \\
\hline FP236240.2 & $\begin{array}{l}\text { F: AGATGTCAACACCGCATTAGAG } \\
\text { R: CCTGCTTTGCCACTTCCTG }\end{array}$ \\
\hline AL392172.1 & $\begin{array}{l}\text { F: TTGTCCTGGATGCCCTCTT } \\
\text { R: CAGACTAGCCAGTCAGTTCTGC }\end{array}$ \\
\hline MSTRG.11733.1 & $\begin{array}{l}\text { F: CCACAGCGATGGCAGATAC } \\
\text { R: GAACCACGGAGCGCAAAT }\end{array}$ \\
\hline AC087269.1 & $\begin{array}{l}\text { F: GGACTGGCAAAAGGAGAAAA } \\
\text { R: CTGGAGCCAAAATGAAGCA }\end{array}$ \\
\hline MSTRG.15047.3 & $\begin{array}{l}\text { F: AAAAGCACCCGTAGTAGCAAA } \\
\text { R: CCAAACTAAAGTAGCCAGCAAG }\end{array}$ \\
\hline AL023581.2 & $\begin{array}{l}\text { F: CCCAACCAATCTGCCTCC } \\
\text { R: CCTCCGATTCACTTCTGTTCTTA }\end{array}$ \\
\hline AC015813.3 & $\begin{array}{l}\text { F: CTCCCTCCTCCCGTATCTG } \\
\text { R: ITTGCTTCGGCTCTGTCTC }\end{array}$ \\
\hline AC008403.3 & $\begin{array}{l}\text { F: AGAGGGCGGTTGTGAGGA } \\
\text { R: ATTAGTCTGTGCCAGGTATCG }\end{array}$ \\
\hline ACTB & $\begin{array}{l}\text { F: CGTGGACATCCGCAAAGA } \\
\text { R: GAAGGTGGACAGCGAGGC }\end{array}$ \\
\hline
\end{tabular}

\section{Results}

Exogenous TTR treatment repressed high glucose induced hRECs tube formation

Human retinal endothelial cells (hRECs) were cultured in $5.5 \mathrm{mM}$ glucose (low group, LG), $25 \mathrm{mM}$ glucose (high glucose, HG), or $25 \mathrm{mM}$ glucose with $4 \mu \mathrm{M}$ TTR (HG + TTR) media. HRECs cellular morphology and physiological nature didn't vary under different treatment conditions as the treatment is for a long time of $48 \mathrm{~h}$ (Additional file 3: Figure S2). Matrigel tube formation assay was conducted to examine TTR impact on the neovascularization. The experiment and analysis workflow was shown in Fig. 1a. Regarding Fig. 1b and c, the tube formation area of HG groups was significantly larger, compared with LG groups (HG average area $=13$, 843.2, LG average area $=9347.6, \mathrm{HG} / \mathrm{LG}=1.48, \quad P<$ 0.001). In HG + TTR co-treatment group, the tube formation area was significantly smaller than HG group $(\mathrm{HG}+\mathrm{TTR}$ average area $=10,121.2, \mathrm{HG}$ average area $=$ 13,843.2, HG + TTR/HG $=0.73, \quad P<0.001$ ), implicating TTR represses hRECs tube formation in a high glucose environment.

\section{Transcriptome sequencing characteristics of genes in glucose and TTR treated hRECs}

The RNA sequencing of each 3 replicate samples in LG, HG and HG + TTR groups identified a total of $566.19 \mathrm{M}$ unique mapped reads with an average length of $139.4 \mathrm{bp}$. In total, we characterized 12,403 known lncRNA transcripts and 146,783 proteincoding transcripts. The transcript length and exon distribution were shown in Fig. 2a. For novel lncRNA prediction, 4 protein coding prediction software PLEK [35], CPAT [36], CNCI [37] and CPC2 [38] were applied and 1184 novel transcripts showed non or low coding capability (Fig. 2b). Based on genomic located with nearby coding genes, we classified all novel lncRNAs into 5 categories: intronic lncRNA, sense lncRNA, antisense lncRNA, directional lncRNA and intergenic lncRNA, in which intronic lncRNAs accounted for $64.2 \%$ (Fig. 2c). Besides transcripts identification, alternative splicing events were analyzed. As shown in Fig. 2d, SE (Skipped exon) was the main alternative splicing type in all 5 types (SE-Skipped exon, A5SS-Alternative 5' splice site, A3SS-Alternative 3' splice site, MXEMutually exclusive exons and RI-Retained intron).

\section{DEGs and TTR-DEGs identification}

Of all the lncRNAs and mRNAs identified, there were a total of 11,407 significantly differentially expressed mRNAs (DE-mRNAs) and 679 significantly differentially expressed lncRNAs (DE-lncRNAs) in HG groups, compared with LG group. Compared with HG group, 


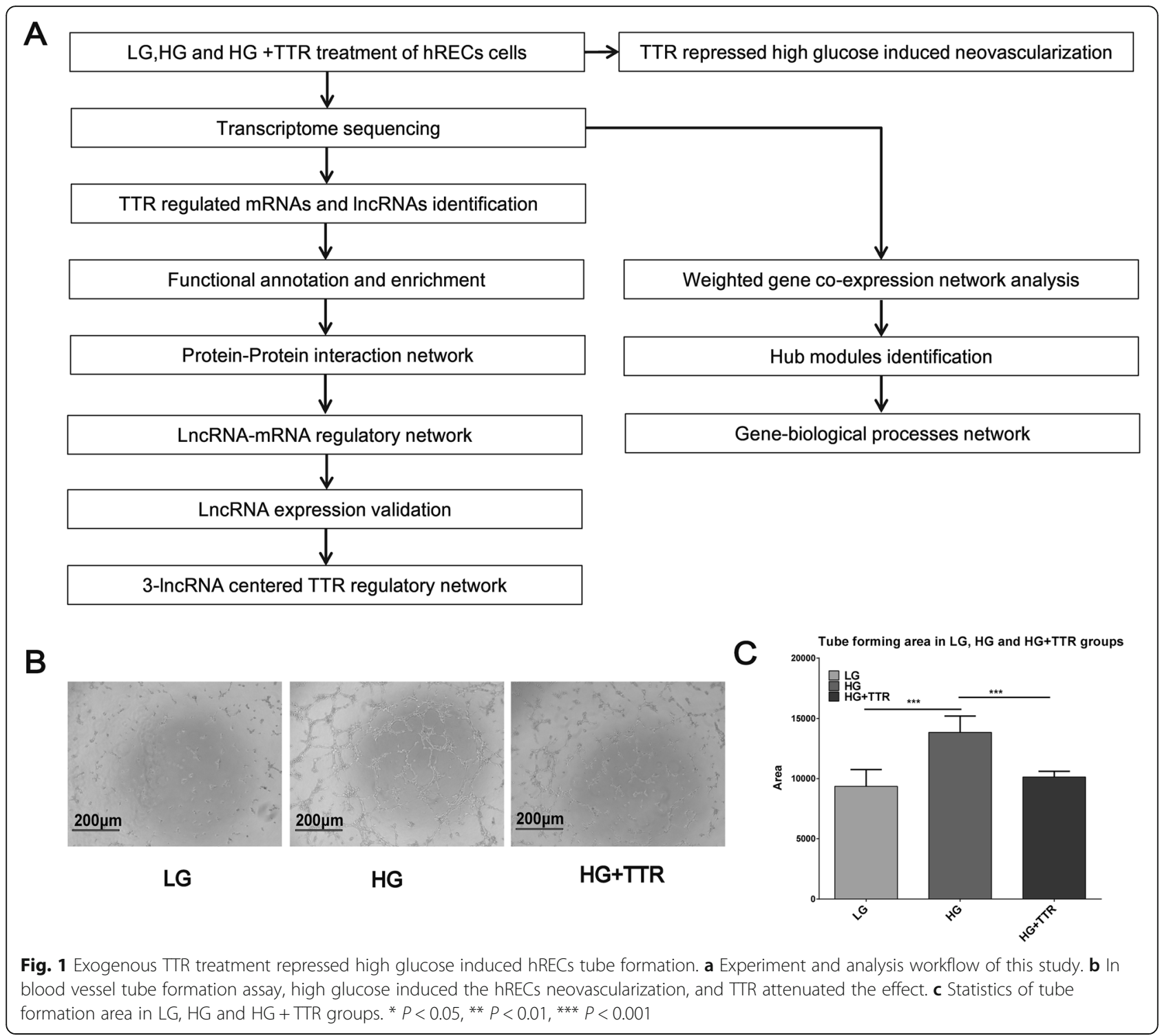

there were 6206 significantly differentially expressed mRNAs (DE-mRNAs) and 194 significantly differentially expressed lncRNAs (DE-lncRNAs) in TTR + HG group. The DE-lncRNAs and DE-mRNAs were shown in volcano plot in Fig. 3a-b. As TTR exerts the protective effects comparing with high glucose, we mainly focused on DEGs with contradictory expression fold-change trend in TTR + HG group versus HG group and HG group versus LG group. A total of 853 mRNAs and 48 lncRNAs meet the criteria of opposite trend and were named as TTR related DEGs (TTR-DEGs), shown in Additional file 1: Table S1. Supervised hierarchical clustering was constructed to show TTR-DEGs expression in 9 samples of 3 groups (Fig. 3c-d). Samples in LG group and HG + TTR group were clustered together and showed similar expression pattern.
KEGG pathway, gene ontology annotation and GSEA analysis

Based on the premise that TTR exert the opposite role of high glucose, functional annotation and enrichment analysis were conducted to explore the role of 853 TTRmRNAs. KEGG pathways analysis enriched pathways like apoptosis, glucagon signaling pathway, cAMP signaling pathway, RNA degradation and transport, MAPK signaling pathway, ErbB signaling pathway and Pyrimidine metabolism (Fig. 4a). The Gene Ontology analysis enriched biological processes like sister chromatid cohesion, chromosome segregation, regulation of cell cycle, positive regulation of $\mathrm{I}-\mathrm{kB}$ kinase/NF- $\mathrm{kB}$ signaling and cellular response to DNA damage stimulus (Fig. 4b). In addition, all mRNA expression levels in LG, HG and HG + TTR groups were applied to conduct GSEA 


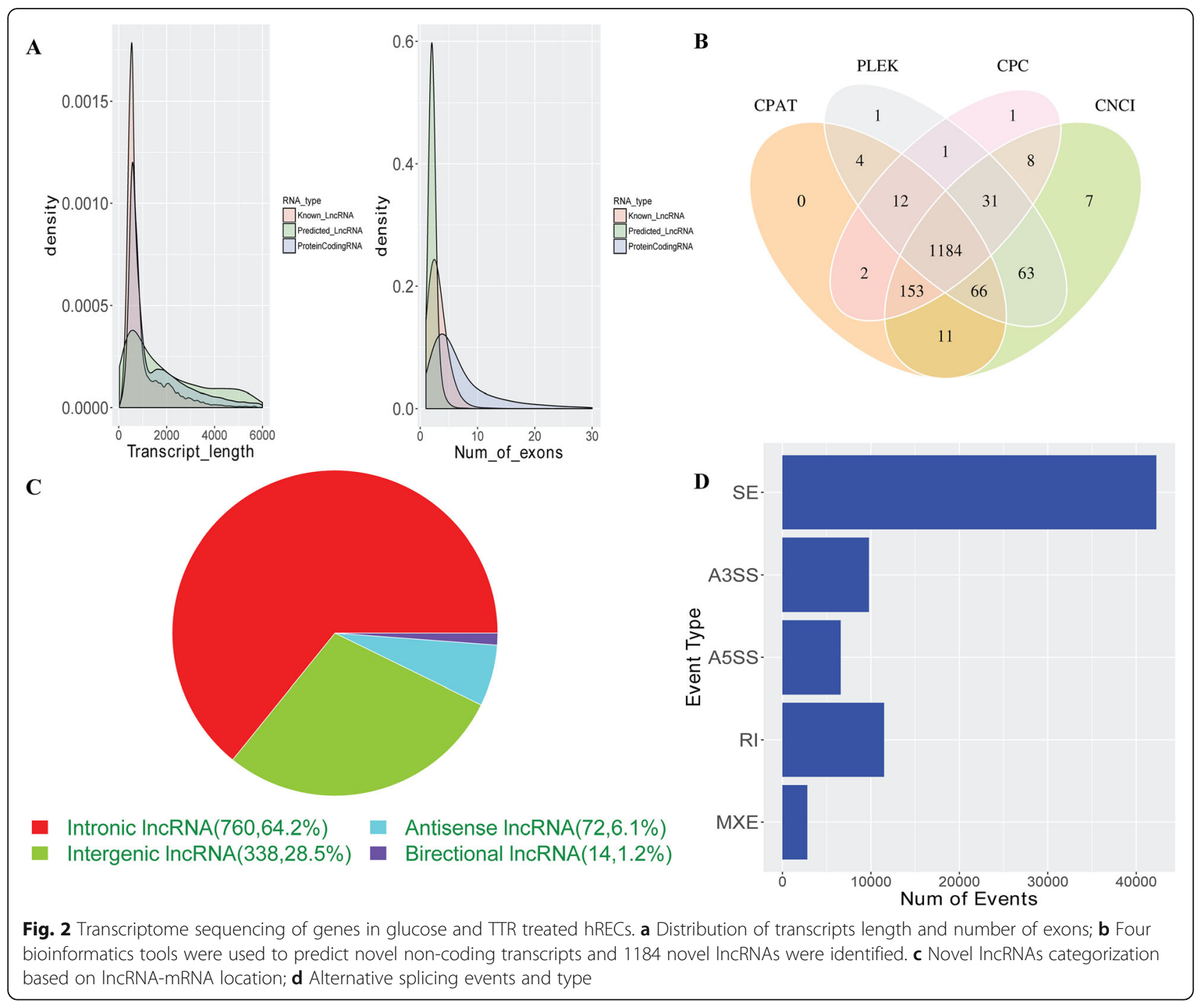

functional analysis. As shown in Fig. 3c, the GSEA analysis of mRNA in HG and LG identified Cell cycle related pathway like DNA replication, and inflammatory pathways like IL2-STAT5 (Fig. 4c). GSEA analysis of mRNA in TTR + HG and HG identified pathways involved in cell cycle like G2/M checkpoint, cell apoptosis pathways like Fas pathway, neovascularization like ERBB1/4 receptor pathway and oxidative stress response like Nitric Oxide Stimulates Guanylate Cyclase, and P38-MKK3 pathway (Fig. 4d).

\section{The TTR protein-protein interaction network and sub- networks function}

To further elucidate the regulatory and interaction relationship, the 813 TTR-mRNAs were imported into the STRING database and visualized in Cytoscape. The STRING database identified a TTR-network of 548 TTR-mRNAs and 2135 edges (interaction relationship). Then the MCODE algorithm was implemented and the
TTR-network was categorized into 5 sub-networks. The biological processes of TTR-mRNAs in each subnetwork were analyzed. The 27 TTR-mRNAs in TTR sub-network 1 were mainly involved in ubiquitin mediated proteolysis and cell cycle. The 19 TTR-mRNAs in TTR sub-network 2 were well known to influence DNA replication and cell cycle. The 39 TTR-mRNAs in subnetwork 3 were reported to regulate mRNA splicing via spliceosome, RNA transport, protein translation and apoptosis mediated by MAPK and NFkB pathway. The 32 TTR-mRNAs in sub-network 4 exert the role of Oxidative phosphorylation regulation. The function of 19 TTR-mRNAs in sub-network 5 was transcriptional regulation and DNA repair (Fig. 5).

\section{WGCNA analysis identified hub modules both regulated by glucose and TTR}

To further identify genes correlated with glucose and TTR treatment, gene expression module-traits (glucose 


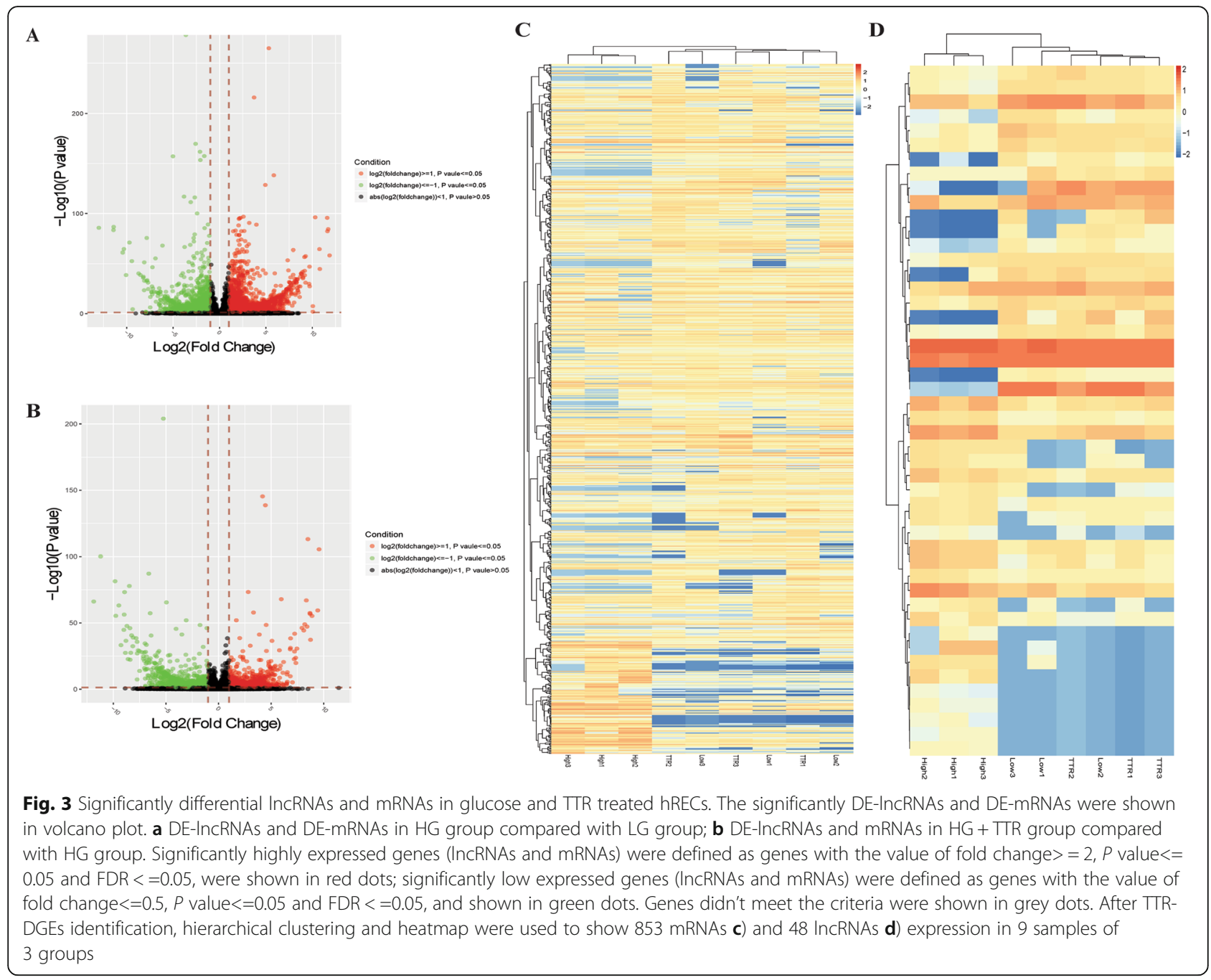

and TTR) relationship were further illustrated with WGCNA analysis. As shown in Fig. 6a, sample clustering based on all gene expression characteristics generated 3 groups, conforming to known LG, HG and HG + TTR grouping. To further identify mRNAs both regulated by glucose and TTR, gene expression modules were calculated, of which module clusters were shown in Fig. 6b. Then the Soft threshold were calculated and set as 10 (Fig. 6c) and a hub module in MEgreen color showed largest Pearson correlation coefficient with both glucose and TTR (0.86 and 0.8, respectively, Fig. $6 \mathrm{~d}$ ). The hub module was comprised of 133 genes, and involved in biological processes like oxidative stress, angiogenesis, MAPK pathway, proliferation and apoptosis (Fig. 6e). The Cytoscape plug-in was used to annotate and visualize the gene-function network of 133 in the hub module. As shown in Fig. 7, genes like CTSA (Cathepsin A) [39], FEZ1 (Fasciculation And Elongation Protein Zeta 1) [40] and HMOX1 (Heme Oxygenase 1) [41] were involved in biological processes like negative regulation of autophagy. Genes like BTG1 (BTG AntiProliferation Factor 1) [42], ABL1 (ABL Proto-Oncogene 1, Non-Receptor Tyrosine Kinase) [43], RRAS (RAS Related) [44], ITGA5 (Integrin Subunit Alpha 5) [45], JAK1 (Janus Kinase 1) [46], ANXA3 (Annexin A3) [47] and HMOX1 [48] were reported to regulate angiogenesis.

\section{LncRNA-mRNA regulatory network based on cis, trans and ceRNA interaction relationship}

To identify lncRNA-mRNAs regulating network in glucose and TTR treated hRECs, IncRNA targets acting in cis, trans and ceRNA way was predicted. In cis regulatory network shown in Fig. 8a, lncRNAs nearby coding mRNAs were chosen for network construction. Edges in the network denote relative upstream or downstream location within $10 \mathrm{~kb}$ in the same chromosome. The cis regulatory network was composed of 33 cis IncRNAmRNA pairs and 61 TTR-DEGs (33 TTR-lncRNAs and 28 TTR-mRNAs) genes of 133 genes. To further explore trans-regulatory network, Pearson correlation coefficient 


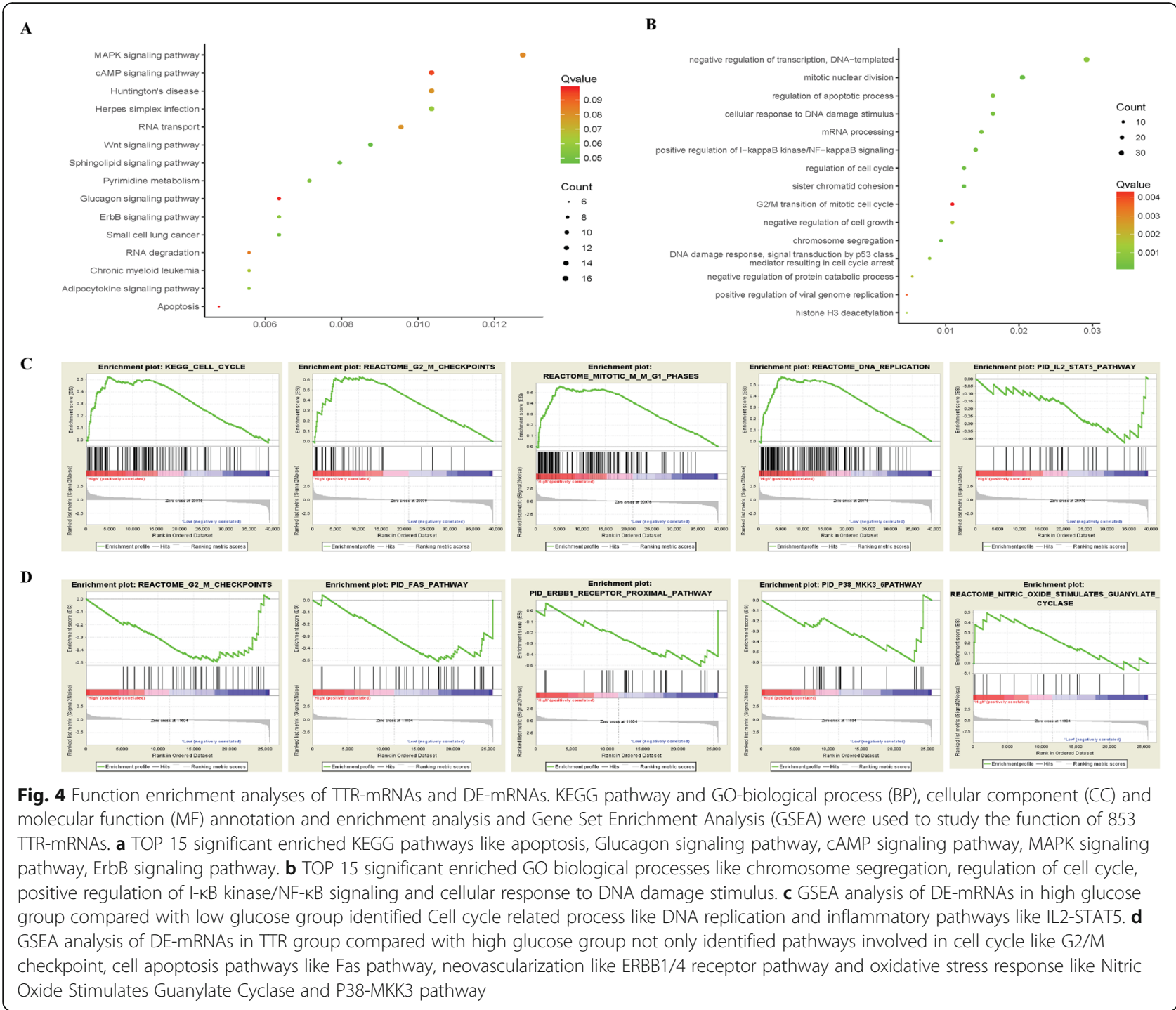

of gene expression were calculated and lncRNA-mRNA coefficient $\geq 0.95$ or $\leq-0.95$ were screened. As shown in Fig. 8b, a network of 96 TTR-DEGs and 184 TTRlncRNA/mRNA pairs was constructed in Cytoscape. In addition to cis and trans regulatory relationship, TTRlncRNAs acting as ceRNAs of TTR-mRNA were predicted based on miRanda database. A total of 12 TTR-lncRNAs, 12 miRNAs and 12 mRNAs constituted 11 TTR-lncRNA/ miRNA/mRNA sub-networks (Fig. 8c).

\section{The $\mathbf{5}$ functional IncRNA-mRNA trans networks}

Integrating 5 mRNA sub-networks in Fig. 4 and lncRNA-mRNA trans network in Fig. 8b, we further constructed 5 functional lncRNA-mRNA networks. 12 TTR-IncRNAs correlated with 11 TTR-mRNAs in a potential trans sub-network 1 were involved in ubiquitin mediated proteolysis and cell cycle (Fig. 9a). 9 TTRlncRNAs correlated with 7 TTR-mRNAs in a potential trans sub-network 2 were involved in DNA replication and cell cycle (Fig. 9b). 12 TTR-lncRNAs correlated with 23 TTR-mRNAs in a potential trans sub-network 3 were involved in mRNA splicing via spliceosome, RNA transport, protein translation and apoptosis mediated by MAPK and NFkB pathway (Fig. 9c). 9 TTR-lncRNAs correlated with 12 TTR-mRNAs in a potential trans sub-network 4 were involved in Oxidative phosphorylation regulation (Fig. 9d). 5 TTR-lncRNAs correlated with 8 TTR-mRNAs in a potential trans sub-network 5 were involved in transcriptional regulation and DNA repair (Fig. 9e).

\section{A 3-IncRNA centered TTR regulatory network with diagnostic performance}

To further pinpoint the regulatory and functional network centered by IncRNAs in TTR and glucose on hRECs, the expression of 10 TTR-IncRNAs with the 


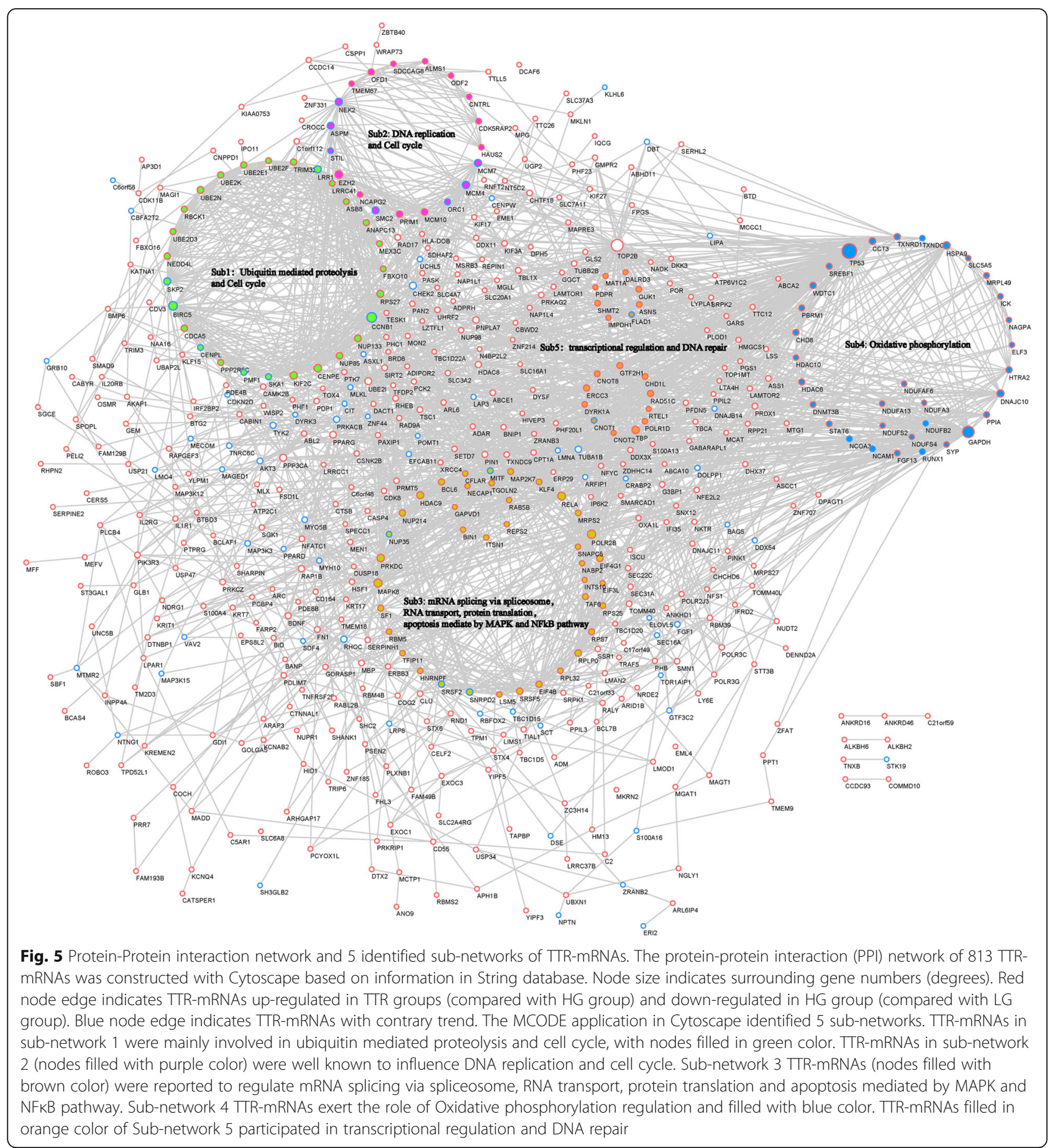

largest node size (indicating largest number of direct surrounding genes in the trans-network), AL023581.2, AL392172.1, FP236240.2, AC105345.1, AC087269.1, AC015813.3, FRMD6-AS2, AC008403.3, MSTRG.15047.3 and MSTRG.11733.1 were validated by qRT-PCR in hRECs. As shown in Fig. 10a, 3 TTR-IncRNAs (MSTRG.15047.3, FRMD6-AS2 and AC008403.3) showed concordant significant expression trend in both RNA-Seq and qRT-PCR detection methods. The predicted novel IncRNA MSTRG.15047.3 in LG and HG + TTR groups was significantly up-regulated by 15.39 fold and 8.11 fold, compared with HG groups, respectively. LncRNA FRMD6-AS2 (FERM domain-containing protein 6 Antisense RNA 2) in LG and HG + TTR groups showed decreased expression of 1.81 and 3.25 fold, compared with HG group, respectively. LncRNA AC008403.3 in LG and 

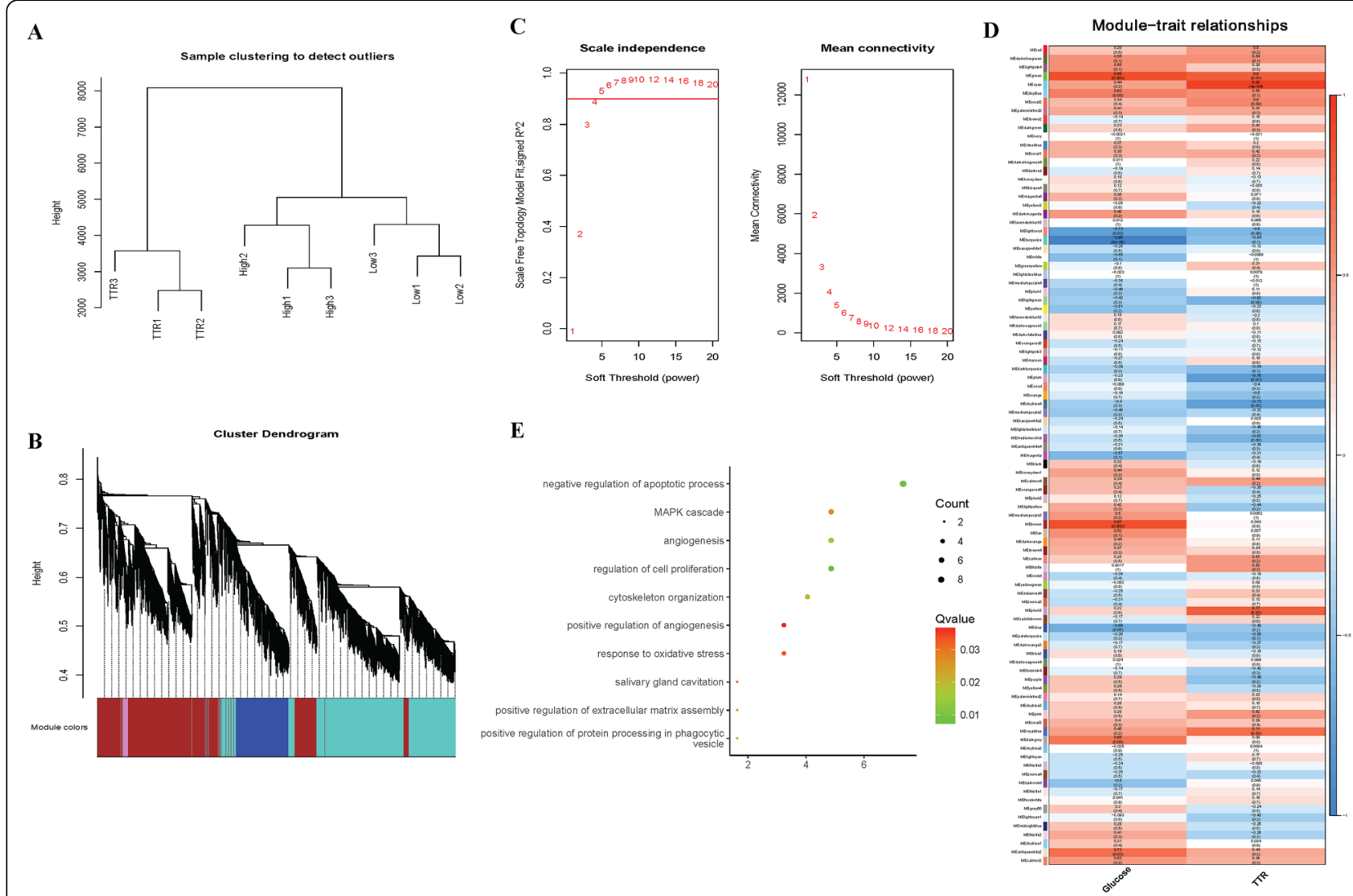

Fig. 6 Weighted gene co-expression network analysis (WGCNA) analysis identified hub modules both regulated by glucose and TTR (a) Sample clustering as clustered to detect samples outliers and (b) cluster dendrogram was calculated; (c) Soft threshold calculation and mean connectivity to identify the soft threshold with best performance; (d) Gene expression module-trait (glucose and TTR) relationships were calculated when the soft threshold was 10; (e) the GO-biological processes of 133 genes in green module. The module genes were involved in biological processes like oxidative stress, angiogenesis, MAPK pathway, proliferation and apoptosis

HG + TTR groups was significantly up-regulated with a fold change of 2.32 and 1.33, compared with HG group, respectively. Then we focused on the 3 TTR-lncRNAs and integrated predicted targets in cis, trans and ceRNA way, and built a 3-lncRNA centered TTR regulatory network (Fig. 10b). Finally, the expression of lncRNA MSTRG.15047.3, AC008403.3 and FRMD6-AS2 was examined in aqueous humor and serum samples from 30 DR patients and 10 normal controls (patients without diabetes). In DR patients, MSTRG.15047.3 and $A C 008403.3$ showed significantly relative higher expression in both aqueous humor and serum samples, compared with normal controls, and FRMD6-AS2 was significantly down-regulated (Fig. 10c), showing promising potential as DR diagnostic biomarkers.

\section{Discussion}

Recently, it was reported that dysfunction of TTR was bound up with system diseases and the intervention of TTR improved multiple clinical manifestations of hereditary transthyretin amyloidosis $[49,50]$. TTR was also involved in various biological processes such as proteolysis, nerve regeneration, autophagy, glucose homeostasis and angiogenesis regulation [51]. In ophthalmic diseases, abnormal TTR expression was reported in high myopia and diabetic retinopathy patients. Exogenous TTR treatment inhibited hRECs proliferation, migration and tube forming capability and induced apoptosis in vitro $[12,13]$. However, the underlying mechanism still remains unknown. In this study, through RNAsequencing, the basic transcriptome characteristics of hRECs underlying hyperglycemia and TTR with high glucose conditions were first determined. Then, through functional annotation, protein-protein interaction and co-expression analysis, the protective role of TTR in hRECs dysfunction including signaling pathways, biological processes and trait modules were further explored. Furthermore, potential targets of TTR related lncRNAs were predicted and 3 coding-non-coding networks were constructed based on putative IncRNA 


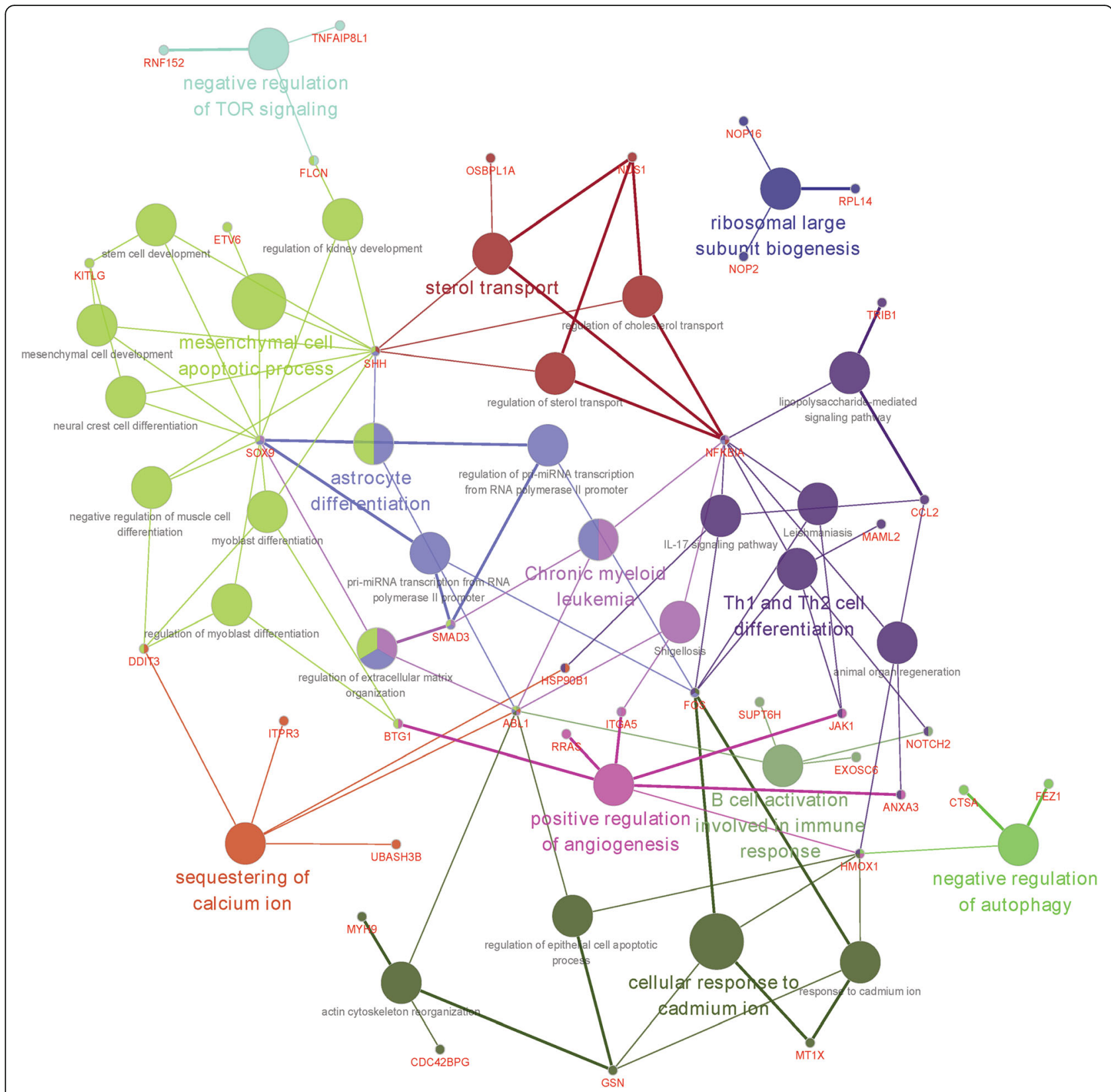

Fig. 7 Gene-biological processes functional network of hub module. Functional analysis and enrichment of 133 genes in hub module was conducted with Cytoscape ClueGo application. Genes like CTSA, FEZ1 and HMOX1 were involved in biological processes like negative regulation of autophagy. Genes like BTG1, ABL1, RRAS, ITGA5, JAK1, ANXA3 and HMOX1 were reported to regulate angiogenesis

acting mode. Finally, a 3-lncRNA centered regulatory hub network with clinical significance was proposed to explain the function of TTR attenuating neovascularization.

The functional annotation of TTR related mRNAs corroborated previous reports of relative decreased expression of TTR in DR patients and neovascularization function. In our study of TTR function in hRECs, biological processes involved in cell proliferation, migration and neovascularization were significantly enriched, in lining with studies that TTR modulated human umbilical vein endothelial cells (HUVECs) apoptosis and inhibit migration, regulation of hRECs apoptosis, proliferation and migration. In addition, biological processes and pathways like response to oxidative stress, inflammatory signaling like IL2-STAT5, and regulation of autophagy were also enriched and reported for the first time. The intricate cross-talking between oxidative stress, inflammatory signaling pathway, autophagy and apoptosis were reported in melatonin ameliorating DR 


\begin{tabular}{|c|c|}
\hline $\begin{array}{l}\text { Fing } \\
\text { Fig. } 8 \text { LncRNA-mRNA regulatory network ba } \\
\text { TTR-DEGs were shown in red color and dowr } \\
\text { expressed. TTR-mRNAs were represented in c } \\
\text { centrality.. Edges in the network denote relat } \\
\text { upstream, blue indicates downstream and ye } \\
m R N A \text { regulatory network. TTR-mRNAs were } \\
\text { network denotes positive or negative correla } \\
\text { Pearson correlation and green edges denote } \\
\text { shown in diamond nodes, TTR-mRNAs in circ }\end{array}$ & 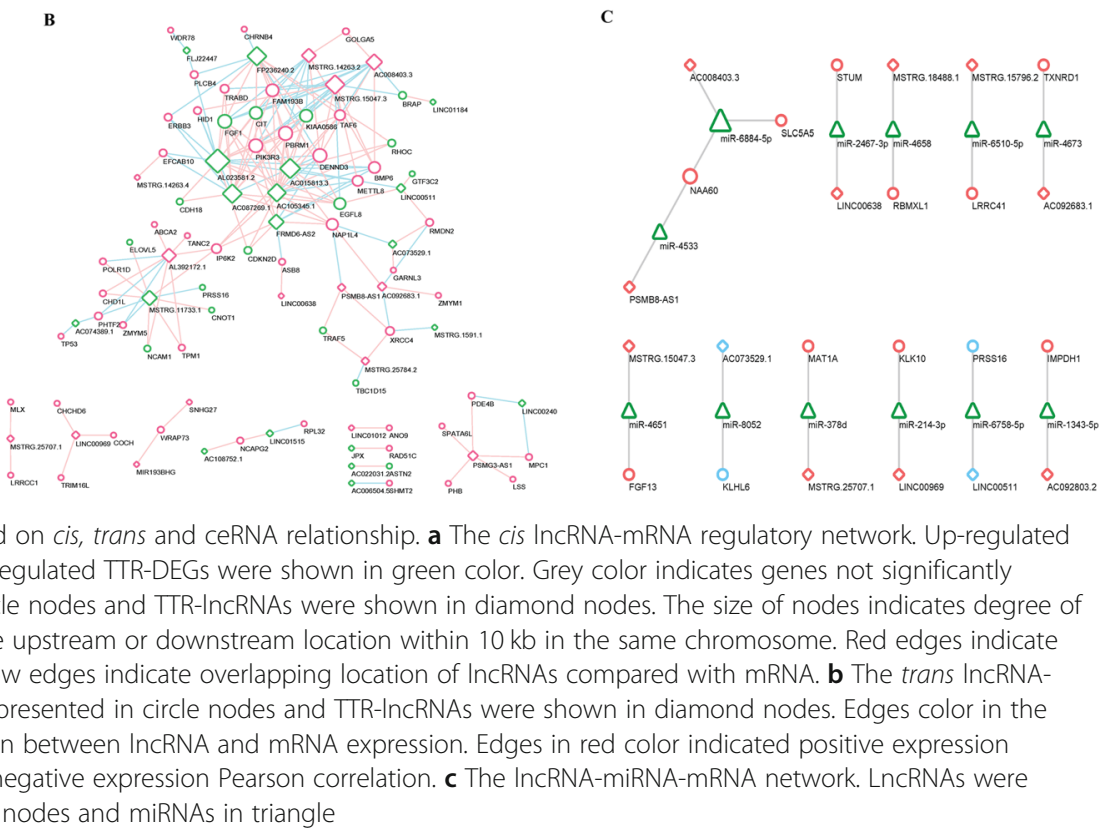 \\
\hline
\end{tabular}

A
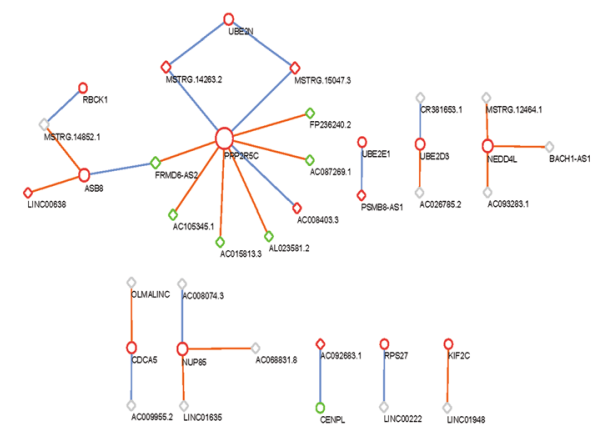

B
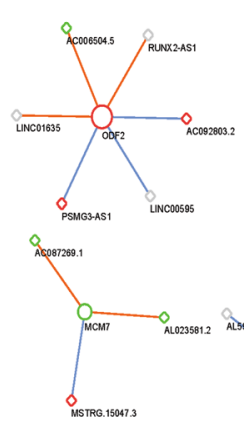

D

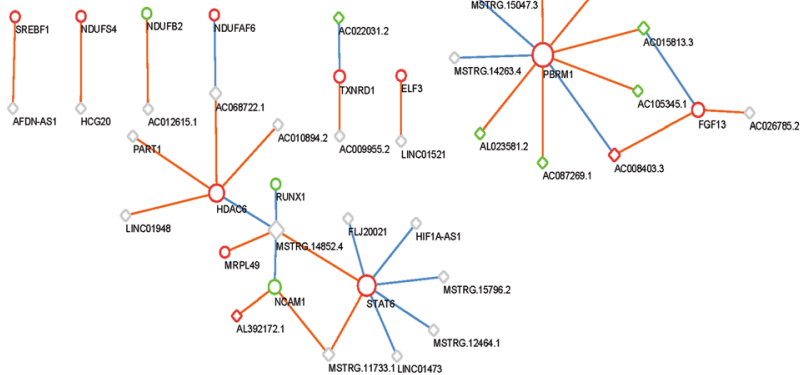

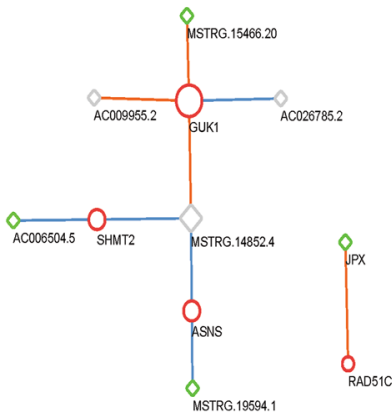

Fig. 9 Five functional IncRNA-mRNA trans regulatory network. a TTR-IncRNAs-mRNA trans regulatory sub-network1: ubiquitin mediated proteolysis and cell cycle; $\mathbf{b}$ TTR-IncRNAs-mRNA trans regulatory sub-network2: DNA replication and cell cycle; $\mathbf{c}$ TTR-IncRNAs-mRNA trans regulatory sub-network3: mRNA splicing via spliceosome, RNA transport, protein translation and apoptosis mediated by MAPK and NFKB pathway; d TTR-IncRNAs-mRNA trans regulatory sub-network4: Oxidative phosphorylation regulation; e TTR-IncRNAs-mRNA trans regulatory sub-network5: transcriptional regulation and DNA repair 
A

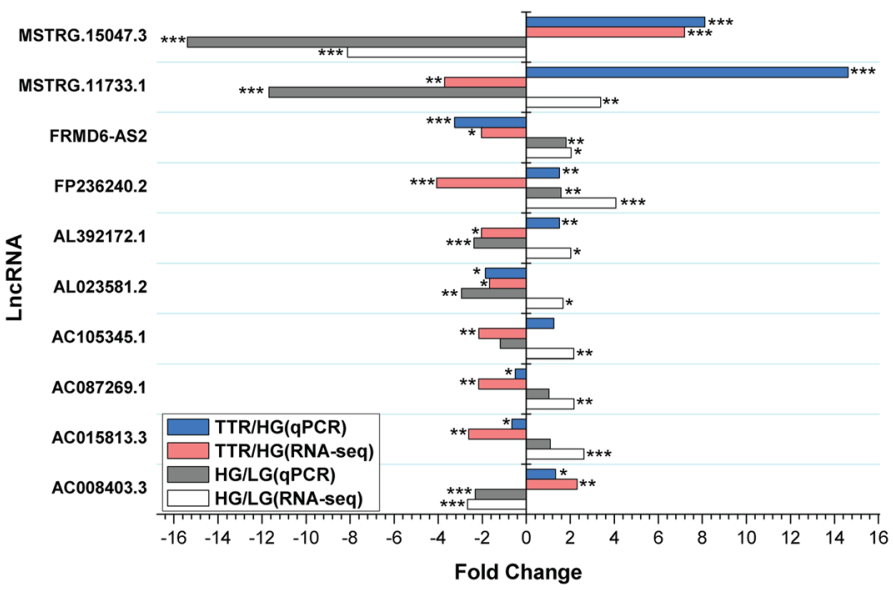

B

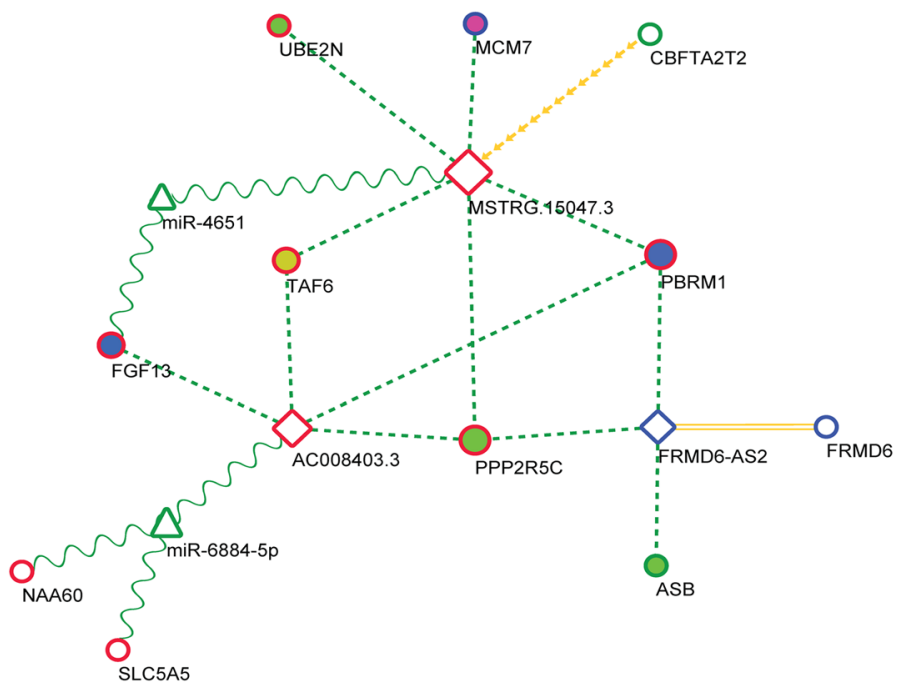

C

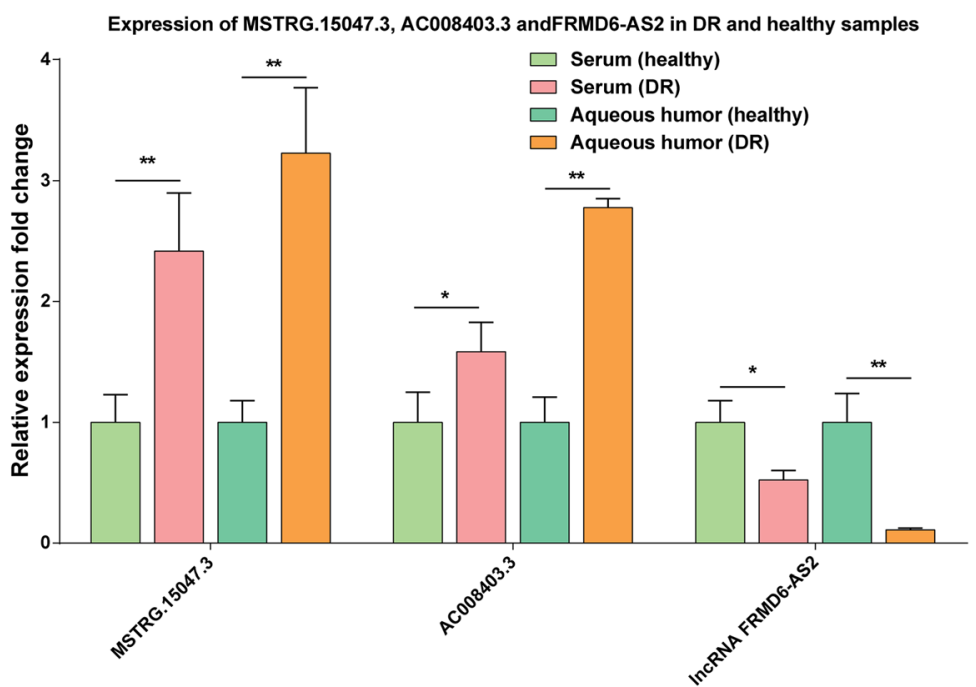

Fig. 10 (See legend on next page.) 
(See figure on previous page.)

Fig. 10 LncRNA expression validation and 3-IncRNA centered TTR regulatory network. a The 10 TTR-IncRNAs expression fold change detected with RNA-Seq and qRT-PCR, respectively. ${ }^{*} P<0.05,{ }^{* *} P<0.01$, ${ }^{* *} P<0.001$. b A 3-IncRNA centered TTR regulatory network. 3 TTR-IncRNAs were shown in diamond nodes, TTR-mRNAs in circle nodes and miRNAs in triangle. Equal dash edges indicate trans relationship. Yellow separate arrow pointing from CBFTA2T2 to MSTRG.15047.3 indicates upstream nearby location. Yellow parallel edges between FRMD6-AS2 and FRMD6 represent overlapping and antisense location. Sinewave edges between TTR-IncRNAs, TTR-mRNAs with miRNAs denotes potential ceRNA relationship. TTRmRNAs filled in green (PPP2R5C, UBE2Nand ASB), purple (MCM7), brown (TAF6) and blue (FGF13 and PBRM1) indicates genes in sub-network 1-4. c The expression of InCRNA MSTRG.15047.3, AC008403.3 and FRMD6-AS2 were examined with qRT-PCR in aqueous humor and serum samples from 30 DR patients and 10 healthy participants. ${ }^{*} P<0.05$, ${ }^{* *} P<0.01$, ${ }^{* *} P<0.001$

progression [52]. Thus, we speculated that there may exist a similar regulatory network, composed of oxidative stress, inflammation signaling, autophagy and apoptosis, linking the protective function of TTR and DR. The 5 protein-protein interaction sub-networks in Fig. 4 implied that TTR might orchestrate pathways in transcriptional and post-transcriptional levels. Genes in subnetwork 1 implied that TTR might regulate cell cycle via ubiquitin mediated proteolysis, and the function of subnetwork 3 suggested that TTR modulates MAPK and NF- $\mathrm{B}$ p pathway through RNA transportation, splicing and translation, post-transcriptionally. In transcriptional level, the function of genes in the sub-network 5 was focused on DNA repair, an important link of oxidative stress, inflammatory signaling, autophagy and apoptosis [53-55]. Hence, the functional annotation provides a comprehensive direction for further research of how TTR rescuing high glucose induced neovascularization abnormality.

Another important part of our work was the investigation of TTR related lncRNAs and their potential regulatory role in DR. As non-coding RNAs, the functional mode of lncRNAs via regulating mRNA expression in cis, trans or as miRNA sponge has been well recognized in angiogenesis and vascular diseases [56]. LncRNA MEG3 knockdown aggravated retinal vessel dysfunction by the activation of PI3k/Akt signaling [16]. Through regulating p38-MAPK pathway, HOTTIP improved diabetic retinal microangiopathy [19]. As an important inflammation regulator, lncRNA MALAT1 was reported to affect retinal endothelial cell proliferation, migration, and tube formation through cross-talking with p38 MAPK signaling pathway [20,21]. As $m i R-29 b$ and $m i R$ 150-5p sponge or VEGF ceRNA, IncRNA MIAT (Myocardial infarction associated transcript) was reported to regulate endothelial cell apoptosis [18]. Another role that well studied in cardiovascular diseases is IncRNA ANRIL (antisense non-coding RNA in the INK4 locus), which regulated VEGF expression and function in diabetic retinopathy via $m i R-200 b$, and $E Z H 2$ (Enhancer of Zeste 2 Polycomb Repressive Complex 2 Subunit) of the PRC2 (Polycomb Repressive Complex 2) complex [23]. However, the role of lncRNAs in TTR ameliorating hRECs dysfunction has not yet been reported. In this paper, a 3 lncRNA-mRNAs regulatory network and 5 lncRNAs-mRNAs sub-networks in cis, trans and ceRNA were constructed for the first time. The networks would contribute to understanding the underlying mechanism of TTR functions in DR from the perspective of different lncRNAs acting mode.

In addition, expression of 10 lncRNAs in lncRNAmRNAs networks were validated by qRT-PCR and 3 lncRNAs including 2 up-regulated novel lncRNAs, i.e., MSTRG.15047.3 and AC008403.3, 1 down-regulated lncRNA, i.e., FRMD6-AS2 (FRMD6 Antisense RNA 2). The 3 lncRNAs formed a complicated potential interacting network, including 11 mRNAs and 2 miRNAs. Based on the network, the novel predicted lncRNA MSTRG.15047.3 might interact with nearby up-located CBFTA2T2 in a cis-regulatory way, trans-regulates cell cycle and neovascularization via $U B E 2 N$ (Ubiquitin Conjugating Enzyme E2 N), PPP2R5C (Protein Phosphatase 2 Regulatory Subunit B' Gamma), MCM7(Minichromosome Maintenance Complex Component 7), TAF6 (The TATA-Box Binding Protein Associated Factor 6) and PBRM1 (Polybromo 1), or just sponge miR-4651 and promote FGF13 (Fibroblast Growth Factor 13) expression. Gene UBE2N encoded a member of the E2 ubiquitin-conjugating enzyme family and was reported to be involved in cell cycle and error-free DNA repair pathway [57]. The UBE2V1 (Ubiquitin Conjugating Enzyme E2 V1)-UBE2N heterodimer acts in concert with TRIM5 (Tripartite Motif Containing 5) to activate the MAP 3 K7/TAK1 complex and induce expression of $\mathrm{NF} K \mathrm{~B}$ and MAPK-responsive inflammatory genes [58, 59]. $P P P 2 R 5 C$ gene belongs to the phosphatase $2 \mathrm{~A}$ regulatory subunit B family. The $P P 2 A$ (Protein Phosphatase 2 Phosphatase Activator) -PPP2R5C holoenzyme may specifically dephosphorylate and activate TP53 (Tumor Protein P53) and play a role in DNA damage-induced inhibition of cell proliferation, as well as regulate the ERK signaling pathway through ERK dephosphorization [60]. MCM7 acts as component of the MCM2-7 complex (MCM complex) and may be involved in the formation of replication forks and in the recruitment of other DNA replication related proteins [61]. The TATA-Box Binding Protein Associated Factor 6 (TAF6) are components of the transcription factor IID (TFIID) complex 
and modulates cell apoptosis in a TP53 dependent [62] and independent [63] way. PBRM1 encodes a subunit of ATP-dependent chromatin-remodeling complexes. In clear cell renal cancer, concomitant loss of PBRM1 rescues VHL-induced replication stress, maintaining cellular fitness and allowing proliferation [64]. Fibroblast Growth Factor 13 possesses broad mitogenic and cell survival activities, including embryonic development, cell growth, morphogenesis and invasion [65]. For AC008403.3, in addition to sharing 4 trans targets of TAF6, PPP2R5C, PBRM1 and FGF13 with MSTRG.15047.3 may act as a ceRNA of NAA6O (N (Alpha)-Acetyltransferase 60, NatF Catalytic Subunit) and SLC5A5 (Solute Carrier Family 5 Member 5) via sponging miR-6884-5p. NAA60 encodes an enzyme that localizes to the Golgi apparatus and transfers an acetyl group to the $\mathrm{N}$-terminus of free proteins [66]. SLC5A5 encodes a member of the sodium glucose cotransporter involved in thyroxine metabolism, a shared molecular function of TTR [67]. As the antisense RNA and potential cis-regulator of FRMD6 (FERM Domain Containing 6), a known hippo pathway kinase regulator [68], lncRNA FRMD6-AS2 might interact with PBRM1, PPP2R5C and ASB (Arylsulfatase B) to regulate cell proliferation and neovascularization. Further studies will be conducted to explore the exact role of 3-IncRNA regulatory network linking TTR and DR neovascularization.

Finally, IncRNAs MSTRG.15047.3, AC008403.3 and FRMD6-AS2 showed significant differential expression levels in aqueous humor and serum samples of DR patients and normal controls (patients without diabetes), made possible 3 lncRNAs as diagnostic biomarkers in DR. Several studies investigated the clinical significance of lncRNAs in DR, like MIAT [17] and MALAT1 [21]. In this study, we proposed 3 of 10 lncRNAs potentially involved in DR progression, and we would explore their function and molecular mechanism in the future. Additionally, the correlation of lncRNAs expression with clinical parameters, and especially performance as DR biomarkers would be studied, with more samples, including healthy donors and other ocular diseases.

In summary, it was concluded that TTR repressed hRECs neovascularization, via a 3-lncRNA regulatory network. This investigation was mainly based on glucose and exogenous TTR treatment of hRECs, RNA sequencing, bioinformatics analysis and qRT-PCR validation in clinical samples. There are several limitations in this study. First, hRECs cell line was used as the DR cell model, and the tube forming assay demonstrated that TTR repressed neovascularization. Whether the neovascularization repressing effect of TTR and 3-IncRNA regulatory network were restricted to hRECs, or represents a general mechanism in microvascular endothelial cells is an important question. In fact, Kun Shan .et al. employed other microvascular endothelial cell lines, like human umbilical vein endothelial cells (HUVECs), EA.hy.926 and human coronary artery endothelial cells (HCAECs) to investigate retinal vascular dysfunction [69]. In addition, using primary human retinal microvascular endothelial cell (HRMECs) would help to illustrate the point. Secondly, this study was conducted in vitro, and investigation of TTR effect in DR animal models, like streptozotocin (STZ) administration, pancreatectomy and genetic models [70] would provide more solid evidence to support the conclusion. Thirdly, an exogenous TTR was employed to examine the function and a vector or virus mediated TTR overexpression approach would provide more details about the endogenous function of TTR. Lastly, we speculated that the 3-IncRNA regulatory network play a pivotal role in TTR function, and more experiments, like rescue assays of 3 lncRNAs knockdown or overexpression in TTR treatment cells, would help to support the point.

\section{Conclusion}

In summary, we investigated (1) the basic characteristics and landscape of RNA transcriptome in TTR and glucose treated hRECs; (2) the function of TTR-mRNAs from signaling pathway, biological process, subnetworks and hub modules; (3) lncRNA-targets networks and functional sub-networks in cis, trans and ceRNA regulatory way; (4) a 3-IncRNA centered hub network that might mediate the comprehensive function of TTR and serve as potential diagnostic biomarkers in DR. The research would pave the way for understanding the complex biology of TTR in DR progression.

\section{Supplementary information}

Supplementary information accompanies this paper at https://doi.org/10. 1186/s12920-019-0596-2.

Additional file 1: Table S1. We listed the TTR-mRNAs and TTR-IncRNAs, respectively. A total of 853 TTR-mRNAs/transcripts and 48 TTR-mRNAs/ transcripts with normalized expression level in low glucose $(L G)$, high glucose $(H G)$ and TTR + TTR co-treated (TTR) groups, relative expression fold change (log2 transformed) with $P$ value and FDR in TTR/HG and HG/LG groups, gene ID and gene symbol were shown.

Additional file 2: Figure S1. Transcript specific primers targeting IncRNAs were designed. As LncRNA AC008403.3 was co-localized with LMTK3 in chromosome 19 and FRMD6-AS2 and FRMD6 were localized in DNA+/- strands, with partial sequences overlapping (Figure S1), transcript specific primers were designed.

Additional file 3: Figure S2. HRECS cellular morphology in different treatment conditions. HRECs cellular morphology and physiological nature didn't vary under different treatment conditions as the treatment is for a long time of $48 \mathrm{~h}$.

\section{Abbreviations}

ABL1: ABL Proto-Oncogene 1, Non-Receptor Tyrosine Kinase; ACTB: BetaActin; Angpt2: Angiopoietin 2; ANRIL: Antisense non-coding RNA in the INK4 locus; ANXA3: Annexin A3; ASB: Arylsulfatase B; BDNF-AS: BDNF Antisense RNA; BTG1: BTG Anti-Proliferation Factor 1; CeRNA: Competing endogenous RNA; CNC nework: Coding-non-coding network; $\mathrm{CNCl}$ : Coding-Non-Coding Index; CPAT: Coding Potential Assessing Tool; CPC2: Coding Potential 
Calculator 2; CTSA: Cathepsin A; DAVID: The Database for Annotation, Visualization and Integrated Discovery; DEGs: Differentially expressed genes; DE-IncRNAs: Differentially expressed long non-coding RNAs; DEmRNAs: Differentially expressed mRNAs; DR: Diabetic retinopathy; EZH2: Enhancer of Zeste 2 Polycomb Repressive Complex 2 Subunit; FDR: False discovering rate; FEZ1: Fasciculation And Elongation Protein Zeta 1; FGF13: Fibroblast Growth Factor 13; FRMD6-AS2: FRMD6 Antisense RNA 2; GAPDH: Glyceraldehyde-3-phosphate dehydrogenase; GO: Gene Ontology; GRP78: Glucose-regulated protein 78; GSEA: Gene Set Enrichment Analysis; HBMEC-2: Human brain microvascular endothelial cell; HCAECs: Human coronary artery endothelial cells; HG + TTR: High glucose with $4 \mu \mathrm{M}$ TTR; HG: High glucose; HMOX1: Heme Oxygenase 1; HOTTIP: HOXA Distal Transcript Antisense RNA; HRECs: Human retinal endothelial cells; HRMECS: Human retinal microvascular endothelial cells; HRPECs: Human retinal pigment epithelial cells; ITGA5: Integrin Subunit Alpha 5; JAK1: Janus Kinase 1; KEGG: Kyoto Encyclopedia of Genes and Genomes; LG: Low glucose; LncRNAs: Long non-coding RNAs; MALAT1: Metastasis Associated Lung Adenocarcinoma Transcript 1; MCM7: Minichromosome Maintenance Complex Component 7; MCODE: Molecular COmplex Detection; MEG3: Maternally Expressed 3; MIAT: Myocardial Infarction Associated Transcript; MRE: miRNA response element; NAA60: N (Alpha)Acetyltransferase 60, NatF Catalytic Subunit; PBRM1: Polybromo 1; PLEK: Predictor of long non-coding RNAs and messenger RNAs based on an improved k-mer scheme; PP2A: Protein Phosphatase 2 Phosphatase Activator; PPI: protein-protein interaction; PRC2: Polycomb Repressive Complex 2; RBP: Retinol-binding protein; RRAS: RAS Related; SLC5A5: Solute Carrier Family 5 Member 5; Sox2-OT: SOX2 Overlapping Transcript; STR: Short Tandem Repeat; STZ: Streptozotocin; TAF6: The TATA-Box Binding Protein Associated Factor 6; TFIID: Transcription factor IID; TP53: Tumor Protein P53; TRIM5: Tripartite Motif Containing 5; TTR: Transthyretin; TTR-DEGs: TTR related DEGs; UBE2V1: Ubiquitin Conjugating Enzyme E2 V1; VEGF2: Vascular Endothelial Growth Factor; VEGFR1: Vascular Endothelial Growth Factor Receptor 1; WGCNA: Weighted gene co-expression network analysis

\section{Acknowledgements}

We thank Genetic Testing Biotechnology (Shanghai, China) for the help of Short Tandem Repeat (STR) analysis of hRECs.

\section{Authors' contributions}

JS carried out the design of the study and drafted the manuscript. YZ carried out RNA-sequencing and bioinformatics analysis. GF carried participated in cell culture and GRT-PCR examination. YX carried performed the statistical analysis and participated in the design of the study. YY conceived of the study, and participated in its design and coordination. All authors read and approved the final manuscript.

\section{Funding}

This work was supported by National Natural Science Foundation of China (81970819), Jiangsu Young Medical Talents (QNRC2016182) and Natural Science Foundation (BK20160053). The funding body did not play any role in the study design and collection, analysis and interpretation of the data and the write-up of the manuscript.

\section{Availability of data and materials}

Transcriptome data have been submitted to GEO repository: GSE117238.

\section{Ethics approval and consent to participate}

Informed consents were signed for all enrolled patients and healthy individuals. This study followed the tenets of the Declaration of Helsinki and the ARVO statement for research involving human subjects and was approved by the Ethics Committee of Nanjing Medical University (2014-62).

\section{Consent for publication}

Not applicable.

\section{Competing interests}

The authors declare that they have no competing interests.

\section{Author details}

'Department of Ophthalmology, Wuxi People's Hospital affiliated to Nanjing Medical University, Wuxi 214023, Jiangsu, China. ${ }^{2}$ Institute of Biochemistry and Cell Biology, Chinese Academy of Sciences, Shanghai 200031, China. ${ }^{3}$ Key Laboratory of Industry Biotechnology, Ministry of Education, School of Biotechnology, Jiangnan University, Wuxi 214122, Jiangsu, China.

Received: 19 December 2018 Accepted: 27 September 2019

Published online: 15 October 2019

\section{References}

1. Hendrick AM, Gibson MV, Kulshreshtha A. Diabetic retinopathy. Prim Care. 2015;42(3):451-64

2. Song P, Yu J, Chan KY, Theodoratou E, Rudan I. Prevalence, risk factors and burden of diabetic retinopathy in China: a systematic review and metaanalysis. J Glob Health. 2018;8(1):010803.

3. Roy S, Jiang JX, Li AF, Kim D. Connexin channel and its role in diabetic retinopathy. Prog Retin Eye Res. 2017:61:35-59.

4. Cohen SR, Gardner TW. Diabetic retinopathy and diabetic macular edema. Dev Ophthalmol. 2016;55:137-46.

5. Krick TW, Bressler NM. Recent clinically relevant highlights from the diabetic retinopathy clinical research network. Curr Opin Ophthalmol. 2018;29(3):199-205.

6. Heng LZ, Comyn O, Peto T, Tadros C, Ng E, Sivaprasad S, et al. Diabetic retinopathy: pathogenesis, clinical grading, management and future developments. Diabet Med. 2013;30(6):640-50.

7. Monaco HL, Rizzi M, Coda A. Structure of a complex of two plasma proteins: transthyretin and retinol-binding protein. Science. 1995;268(5213): 1039-41.

8. Shao J, Xin Y, Li R, Fan Y. Vitreous and serum levels of transthyretin (TTR) in high myopia patients are correlated with ocular pathologies. Clin Biochem. 2011;44(8-9):681-5.

9. Shao J, Yao Y. Negative effects of transthyretin in high myopic vitreous on diabetic retinopathy. Int J Ophthalmol. 2017;10(12):1864-9.

10. Shao J, Yao Y. Transthyretin represses neovascularization in diabetic retinopathy. Mol Vis. 2016;22:1188-97.

11. Shao J, Yao Y. Repression of retinal microvascular endothelial cells by transthyretin under simulated diabetic retinopathy conditions. Int J Ophthalmol. 2016;9(6):809-15.

12. Shao J, Yin $Y$, Yin $X$, Ji L, Xin Y, Zou J, et al. Transthyretin exerts proapoptotic effects in human retinal microvascular endothelial cells through a GRP78-dependent pathway in diabetic retinopathy. Cell Physiol Biochem. 2017:43(2):788-800.

13. Kopp F, Mendell JT. Functional classification and experimental dissection of long noncoding RNAs. Cell. 2018;172(3):393-407.

14. Thomson DW, Dinger ME. Endogenous microRNA sponges: evidence and controversy. Nat Rev Genet. 2016;17(5):272-83.

15. Yan B, Tao ZF, Li XM, Zhang H, Yao J, Jiang Q. Aberrant expression of long noncoding RNAs in early diabetic retinopathy. Invest Ophthalmol Vis Sci. 2014:55(2):941-51.

16. Qiu GZ, Tian W, Fu HT, Li CP, Liu B. Long noncoding RNA-MEG3 is involved in diabetes mellitus-related microvascular dysfunction. Biochem Biophys Res Commun. 2016:471(1):135-41.

17. Zhang J, Chen M, Chen J, Lin S, Cai D, Chen C, et al. Long non-coding RNA MIAT acts as a biomarker in diabetic retinopathy by absorbing miR-29b and regulating cell apoptosis. Biosci Rep. 2017;37(2):1-10.

18. Yan B, Yao J, Liu JY, Li XM, Wang XQ, Li YJ, et al. IncRNA-MIAT regulates microvascular dysfunction by functioning as a competing endogenous RNA. Circ Res. 2015;116(7):1143-56.

19. Sun Y, Liu YX. LnCRNA HOTTIP improves diabetic retinopathy by regulating the p38-MAPK pathway. Eur Rev Med Pharmacol Sci. 2018;22(10):2941-8.

20. Biswas S, Thomas AA, Chen S, Aref-Eshghi E, Feng B, Gonder J, et al. MALAT1: an epigenetic regulator of inflammation in diabetic retinopathy. Sci Rep. 2018;8(1):6526.

21. Liu JY, Yao J, Li XM, Song YC, Wang XQ, Li YJ, et al. Pathogenic role of IncRNA-MALAT1 in endothelial cell dysfunction in diabetes mellitus. Cell Death Dis. 2014;5:e1506.

22. Li CP, Wang SH, Wang WQ, Song SG, Liu XM. Long noncoding RNA-Sox2OT knockdown alleviates diabetes mellitus-induced retinal ganglion cell (RGC) injury. Cell Mol Neurobiol. 2017;37(2):361-9.

23. Thomas AA, Feng B, Chakrabarti S. ANRIL: a regulator of VEGF in diabetic retinopathy. Invest Ophthalmol Vis Sci. 2017:58(1):470-80.

24. Li Y, Xu F, Xiao H, Han F. Long noncoding RNA BDNF-AS inversely regulated BDNF and modulated high-glucose induced apoptosis in human retinal pigment epithelial cells. J Cell Biochem. 2018;119(1):817-23. 
25. Zhao S, Li T, Li J, Lu Q, Han C, Wang N, et al. miR-23b-3p induces the cellular metabolic memory of high glucose in diabetic retinopathy through a SIRT1-dependent signalling pathway. Diabetologia. 2016;59(3):644-54.

26. Mortuza R, Feng B, Chakrabarti S. miR-195 regulates SIRT1-mediated changes in diabetic retinopathy. Diabetologia. 2014;57(5):1037-46.

27. Nunes RJ, de Oliveira P, Lages A, Becker JD, Marcelino P, Barroso E, et al. Transthyretin proteins regulate angiogenesis by conferring different molecular identities to endothelial cells. J Biol Chem. 2013;288(44):31752-60.

28. Zerbino DR, Birney E. Velvet: algorithms for de novo short read assembly using de Bruijn graphs. Genome Res. 2008;18(5):821-9.

29. Benjamini Y, Hochberg Y. Controlling the false discovery rate: a practical and powerful approach to multiple testing. JR Statist Soc B. 1995;57:289-300.

30. Subramanian A, Tamayo P, Mootha VK, Mukherjee S, Ebert BL, Gillette MA, et al. Gene set enrichment analysis: a knowledge-based approach for interpreting genome-wide expression profiles. Proc Natl Acad Sci U S A. 2005;102(43):15545-50

31. Scardoni $G$, Petterlini M, Laudanna C. Analyzing biological network parameters with CentiScaPe. Bioinformatics. 2009;25(21):2857-9.

32. Langfelder P, Horvath S. WGCNA: an R package for weighted correlation network analysis. BMC Bioinformatics. 2008;9:559.

33. Bindea G, Galon J, Mlecnik B. CluePedia Cytoscape plugin: pathway insights using integrated experimental and in silico data. Bioinformatics. 2013;29(5):661-3.

34. Suarez S, McCollum GW, Jayagopal A, Penn JS. High glucose-induced retinal Pericyte apoptosis depends on association of GAPDH and Siah 1. J Biol Chem. 2015;290(47):28311-20.

35. Li A, Zhang J, Zhou Z. PLEK: a tool for predicting long non-coding RNAs and messenger RNAs based on an improved k-mer scheme. BMC Bioinformatics. 2014;15:311.

36. Wang L, Park HJ, Dasari S, Wang S, Kocher JP, Li W. CPAT: coding-potential assessment tool using an alignment-free logistic regression model. Nucleic Acids Res. 2013;41(6):e74

37. Sun L, Luo H, Bu D, Zhao G, Yu K, Zhang C, et al. Utilizing sequence intrinsic composition to classify protein-coding and long non-coding transcripts. Nucleic Acids Res. 2013:41(17):e166

38. Kang YJ, Yang DC, Kong L, Hou M, Meng YQ, Wei L, et al. CPC2: a fast and accurate coding potential calculator based on sequence intrinsic features. Nucleic Acids Res. 2017;45(W1):W12-W6.

39. Cuervo AM, Mann L, Bonten EJ, d'Azzo A, Dice JF. Cathepsin A regulates chaperone-mediated autophagy through cleavage of the lysosomal receptor. EMBO J. 2003;22(1):47-59.

40. McKnight NC, Jefferies HB, Alemu EA, Saunders RE, Howell M, Johansen T, et al. Genome-wide siRNA screen reveals amino acid starvation-induced autophagy requires SCOC and WAC. EMBO J. 2012;31(8):1931-46.

41. Singh N, Kansal P, Ahmad Z, Baid N, Kushwaha H, Khatri N, et al. Antimycobacterial effect of IFNG (interferon gamma)-induced autophagy depends on HMOX1 (heme oxygenase 1)-mediated increase in intracellular calcium levels and modulation of PPP3/calcineurin-TFEB (transcription factor EB) axis. Autophagy. 2018;14(6):972-91.

42. Iwai K, Hirata K, Ishida T, Takeuchi S, Hirase T, Rikitake Y, et al. An antiproliferative gene BTG1 regulates angiogenesis in vitro. Biochem Biophys Res Commun. 2004;316(3):628-35.

43. Raimondi C, Fantin A, Lampropoulou A, Denti L, Chikh A, Ruhrberg C. Imatinib inhibits VEGF-independent angiogenesis by targeting neuropilin 1dependent ABL1 activation in endothelial cells. J Exp Med. 2014;211(6): 1167-83.

44. Komatsu M, Ruoslahti E. R-Ras is a global regulator of vascular regeneration that suppresses intimal hyperplasia and tumor angiogenesis. Nat Med. 2005 11(12):1346-50.

45. Stenzel D, Lundkvist A, Sauvaget D, Busse M, Graupera M, van der Flier A, et al. Integrin-dependent and -independent functions of astrocytic fibronectin in retinal angiogenesis. Development. 2011;138(20):4451-63.

46. Wang T, Liu J, Xiao XQ. Cantharidin inhibits angiogenesis by suppressing VEGF-induced JAK1/STAT3, ERK and AKT signaling pathways. Arch Pharm Res. 2015;38(2):282-9.

47. Park JE, Lee DH, Lee JA, Park SG, Kim NS, Park BC, et al. Annexin A3 is a potential angiogenic mediator. Biochem Biophys Res Commun. 2005 337(4):1283-7

48. Kim JH, Lee KS, Lee DK, Kim J, Kwak SN, Ha KS, et al. Hypoxia-responsive microRNA-101 promotes angiogenesis via heme oxygenase-1/vascular endothelial growth factor axis by targeting cullin 3. Antioxid Redox Signal. 2014;21(18):2469-82.

49. Benson MD, Waddington-Cruz M, Berk JL, Polydefkis M, Dyck PJ, Wang AK, et al. Inotersen treatment for patients with hereditary transthyretin amyloidosis. N Engl J Med. 2018;379(1):22-31.

50. Adams D, Gonzalez-Duarte A, O'Riordan WD, Yang CC, Ueda M, Kristen AV, et al. Patisiran, an RNAi therapeutic, for hereditary transthyretin amyloidosis. N Engl J Med. 2018;379(1):11-21.

51. Prapunpoj $P$, Leelawatwattana L. Evolutionary changes to transthyretin: structure-function relationships. FEBS J. 2009;276(19):5330-41.

52. Dehdashtian E, Mehrzadi S, Yousefi B, Hosseinzadeh A, Reiter RJ, Safa M, et al. Diabetic retinopathy pathogenesis and the ameliorating effects of melatonin; involvement of autophagy, inflammation and oxidative stress. Life Sci. 2018;193:20-33.

53. Kowluru RA, Kowluru A, Mishra M, Kumar B. Oxidative stress and epigenetic modifications in the pathogenesis of diabetic retinopathy. Prog Retin Eye Res. 2015:48:40-61.

54. Mishra M, Kowluru RA. Retinal mitochondrial DNA mismatch repair in the development of diabetic retinopathy, and its continued progression after termination of hyperglycemia. Invest Ophthalmol Vis Sci. 2014;55(10):6960-7.

55. Tewari S, Santos JM, Kowluru RA. Damaged mitochondrial DNA replication system and the development of diabetic retinopathy. Antioxid Redox Signal. 2012;17(3):492-504

56. Yu B, Wang S. Angio-LncRs: LncRNAs that regulate angiogenesis and vascular disease. Theranostics. 2018;8(13):3654-75.

57. Marteijn JA, Bekker-Jensen S, Mailand N, Lans H, Schwertman P, Gourdin $\mathrm{AM}$, et al. Nucleotide excision repair-induced $\mathrm{H} 2 \mathrm{~A}$ ubiquitination is dependent on MDC1 and RNF8 and reveals a universal DNA damage response. J Cell Biol. 2009;186(6):835-47.

58. Yamazaki K, Gohda J, Kanayama A, Miyamoto Y, Sakurai H, Yamamoto M, et al. Two mechanistically and temporally distinct NF-kappaB activation pathways in IL-1 signaling. Sci Signal. 2009;2(93):ra66.

59. Fukushima T, Matsuzawa S, Kress CL, Bruey JM, Krajewska M, Lefebvre S, et al. Ubiquitin-conjugating enzyme Ubc13 is a critical component of TNF receptor-associated factor (TRAF)-mediated inflammatory responses. Proc Natl Acad Sci U S A. 2007;104(15):6371-6.

60. Li HH, Cai X, Shouse GP, Piluso LG, Liu X. A specific PP2A regulatory subunit, B56gamma, mediates DNA damage-induced dephosphorylation of p53 at Thr55. EMBO J. 2007;26(2):402-11.

61. Lei M, Tye BK. Initiating DNA synthesis: from recruiting to activating the MCM complex. J Cell Sci. 2001;114(Pt 8):1447-54.

62. Wilhelm E, Kornete M, Targat B, Vigneault-Edwards J, Frontini M, Tora L, et al. TAF6delta orchestrates an apoptotic transcriptome profile and interacts functionally with p53. BMC Mol Biol. 2010;11:10.

63. Wilhelm E, Pellay FX, Benecke A, Bell B. TAF6delta controls apoptosis and gene expression in the absence of p53. PLoS One. 2008;3(7):e2721.

64. Espana-Agusti J, Warren A, Chew SK, Adams DJ, Matakidou A. Loss of PBRM1 rescues $\mathrm{VHL}$ dependent replication stress to promote renal carcinogenesis. Nat Commun. 2017:8(1):2026.

65. Wu QF, Yang L, Li S, Wang Q, Yuan XB, Gao X, et al. Fibroblast growth factor 13 is a microtubule-stabilizing protein regulating neuronal polarization and migration. Cell. 2012;149(7):1549-64.

66. Aksnes H, Goris M, Stromland O, Drazic A, Waheed Q, Reuter N, et al. Molecular determinants of the N-terminal acetyltransferase Naa60 anchoring to the Golgi membrane. J Biol Chem. 2017;292(16):6821-37.

67. Eskalli Z, Achouri Y, Hahn S, Many MC, Craps J, Refetoff S, et al. Overexpression of interleukin-4 in the thyroid of transgenic mice upregulates the expression of Duox 1 and the anion transporter pendrin. Thyroid. 2016;26(10):1499-512.

68. Angus L, Moleirinho S, Herron L, Sinha A, Zhang X, Niestrata M, et al. Willin/ FRMD6 expression activates the hippo signaling pathway kinases in mammals and antagonizes oncogenic YAP. Oncogene. 2012;31(2):238-50.

69. Shan K, Liu C, Liu BH, Chen X, Dong R, Liu X, et al. Circular noncoding RNA HIPK3 mediates retinal vascular dysfunction in diabetes mellitus. Circulation. 2017;136(17):1629-42.

70. Olivares AM, Althoff $K$, Chen GF, Wu S, Morrisson MA, DeAngelis MM, et al. Animal models of diabetic retinopathy. Curr Diab Rep. 2017;17(10):93.

\section{Publisher's Note}

Springer Nature remains neutral with regard to jurisdictional claims in published maps and institutional affiliations. 